JOURNAL OF

SYMPLECTIC GEOMETRY

Volume 1, Number 2, 369-425, 2002

\title{
A h-principle for open relations invariant under foliated isotopies
}

\author{
MÉLANIE BERTELSON ${ }^{1}$
}

\begin{abstract}
This paper presents a natural extension to foliated spaces of the following result due to Gromov: the h-principle for open, invariant differential relations is valid on open manifolds. The definition of openness for foliated spaces adopted here involves a certain type of Morse functions. Consequences concerning the problem of existence of regular Poisson structures, the original motivation for this work, are presented.
\end{abstract}

\section{Introduction.}

Gromov proved in [9] the following theorem.

Theorem 0.1. On an open manifold, the parametric h-principle for open, invariant differential relations is valid.

Heuristically, a differential relation on a manifold $M$ is a differential constraint on the sections of a certain bundle $\pi: E \rightarrow M$. More precisely, it is a subset $\Omega$ of some jet bundle $J^{k}(E)$ of local sections of $E$. A section of $E$ whose $k$-jet extension is entirely contained in the relation satisfies the constraint; it is called a solution of the differential relation $\Omega$. A relation $\Omega$ is said to be open (respectively invariant) when $\Omega$ is an open subset of $J^{k}(E)$ (respectively when isotopies of $M$ can be lifted to isotopies of $J^{k}(E)$ that preserve $\Omega$ ). Letting $\operatorname{Sol}(\Omega)$ (respectively $\Gamma(\Omega)$ ) denote the set of solutions (respectively sections) of $\Omega$, endowed with the weak $C^{k}$ (respectively $C^{0}$ ) topology, the relation $\Omega$ is said to satisfy the parametric h-principle if the $k$-jet extension map

$$
j^{k}: \operatorname{Sol}(\Omega) \rightarrow \Gamma(\Omega): f \mapsto j^{k} f
$$

\footnotetext{
${ }^{1}$ This work has been supported by an Alfred P. Sloan Dissertation Fellowship.
} 
is a weak homotopy equivalence. This means that the map $j^{k}$ induces a bijection inbetween arcwise connected components of $\operatorname{Sol}(\Omega)$ and $\Gamma(\Omega)$, and isomorphisms of the homotopy groups for the various components.

Theorem 0.1 has a lot of important corollaries concerning the existence and classification problems of various types of objects in differential topology and geometry (cf. [10]). For instance, applying Theorem 0.1 to the relation $\Omega^{\mathcal{S}}=\left\{j^{1} \alpha(x) \in J^{1}\left(T^{*} M\right) ; d \alpha(x)\right.$ is nondegenerate $\}$ yields the following result.

Corollary 0.2. Let $M$ be an open manifold. The inclusion of the space of exact symplectic forms on $M$ into the space of nondegenerate 2-forms is a weak homotopy equivalence.

In particular, on an open manifold, existence of a symplectic structure depends only on existence of a nondegenerate 2-form, a problem that belongs to obstruction theory.

The hypothesis that $M$ is open is crucial. Indeed, already for the symplectic relation $\Omega^{\mathcal{S}}$, the h-principle is far from being valid on a closed manifold. In addition to a nondegenerate 2 -form, a closed symplectic manifold admits a de Rham class in $H^{2}(M)$ whose top exterior power does not vanish. More subtle conditions, involving Seiberg-Witten invariants, have been discovered by Taubes (cf. [20]). Furthermore, even when the manifold admits a symplectic structure, not any nondegenerate 2 -form may be deformed into a symplectic form. In general, if every open, invariant differential relation defined on a manifold $M$ satisfies the h-principle, then $M$ must be open, as such a relation can be constructed that admits sections and whose solutions are functions without local maxima.

Motivated by the problem of existence of leafwise symplectic structures on foliated spaces (cf. [1, 2]), we searched for a generalization of Theorem 0.1 to foliated invariant differential relations, that is, differential relations that are invariant under isotopies that preserve a certain foliation on the manifold. This requires finding a good notion of "openness" for foliated spaces. It is important to observe that one may not, in general, impose on the solutions constructed in the proof of Theorem 0.1 to be nicely behaved at infinity. In contrast, a solution of a differential relation (a symplectic structure for instance) on a nonclosed leaf of a foliation that is the restriction of a global solution (a leafwise symplectic structure) is most likely very constrained at infinity, partly due to recurrence phenomena, partly due to the influence of neighboring leaves. The foliated case lies, in some sense, midway 
between the open case and the closed case. Examples of foliations have been exhibited in $[1,2]$ that carry leafwise nondegenerate 2 -forms, and in some case display no identifiable closedness feature, but do not carry any leafwise symplectic form.

On the other hand, open manifolds are characterized by the existence of a positive, proper Morse function, without any local maximum. The proof of Theorem 0.1 suggests to base a definition of openness for foliated manifolds on that characterization. This justifies the following definition.

Definition 0.3. A foliated manifold $(M, \mathcal{F})$ is said to be uniformely open if there exists a smooth function $f: M \rightarrow[0, \infty)$ that has the following properties:

a) $f$ is proper,

b) $f$ has no leafwise local maxima,

c) $f$ is $\mathcal{F}$-generic (cf. Definition 3.22 below).

With this definition of openness, the following result holds:

Theorem 0.4. On a uniformely open foliated manifold, any open, foliated invariant differential relation satisfies the parametric h-principle.

Corollary 0.5. Let $(M, \mathcal{F})$ be a uniformely open foliated manifold. Any leafwise nondegenerate 2-form is homotopic, in the class of leafwise nondegenerate 2-forms, to a leafwise symplectic form.

The leaves of a uniformely open foliation are necessarily open manifolds, but this condition is not sufficient. It can be checked directly (i.e. without quoting Corollary 0.5) that the foliated manifolds introduced in $[1,2]$ do not support any function $f$ satisfying $a$ ) and $b$ ).

The proof of Theorem 0.4 involves consideration of the trajectories of a leafwise gradient vector field for $f$, as did the proof of Theorem 0.1. There are new difficulties. First, the leafwise critical points of $f$ are not isolated but come in families. Thus they cannot be handled one at a time (as they are in the nonfoliated case). Secondly, leafwise critical points may be degenerate, even generically. The set of trajectories converging to a degenerate critical point is not in general well understood. To overcome the latter difficulty one needs to carry out a careful construction of a Riemannian metric for which the trajectories of the associated leafwise gradient vector field are somewhat controlled near the singular locus. 
This paper is organized as follows. Section 1 is a brief introduction to the theory of h-principles (see [10]). Section 2 presents an outline of the proof of Theorem 0.4, and should serve as a reading-guide for the remainder of the text. Section 3 is devoted to making precise the term $\mathcal{F}$-generic referred to in Definition 0.3 , and to introducing the notion of strong $\mathcal{F}$-genericity needed later. Section 4 exhibits some properties of leafwise gradient vector fields (Definition 4.1) of strongly $\mathcal{F}$-generic functions. Section 5 describes the construction of a nice Riemannian metric associated to a strongly $\mathcal{F}$ generic function. The proof of Theorem 0.4 is completed in Section 6. Some examples of uniformely open foliated manifolds are presented in Section 7 . Finally, Section 8 is concerned with Corollary 0.5.

Acknowledgements. I wish to thank Emmanuel Giroux, who suggested to generalize Gromov's result, Alan Weinstein, whose insightful advice has helped this work reach its final shape, and Yasha Eliashberg for many very stimulating conversations. I would like to aknowledge the hospitality of the Max-Planck-Institut für Mathematik (Bonn), where the redaction of this text was carried out. I am grateful to the referee for his relevant and interesting suggestions.

\section{H-principles.}

We state here the definitions and results of the theory of h-principles (cf. [10]) that will be needed in the remainder of the text. We have followed Emmanuel Giroux's beautiful (as yet unpublished) lecture notes [7]. The proofs are reproduced in [1].

\subsection{Differential relations and h-principles.}

Consider a locally trivial fibration $\pi: E \rightarrow M$ with fiber a manifold $F$. Let $E^{k}$ denote the set of $k$-jets of local sections of $\pi: E \rightarrow M$. If $f: U \subset M \rightarrow E$ is a local section defined on an open subset $U$ of $M$, the $k$-jet of $f$ at $x \in U$ is denoted by $j^{k} f(x)$. The set $E^{k}$, endowed with the natural projection $E^{k} \rightarrow M: j^{k} f(x) \mapsto x$, is a locally trivial fibration. For $k \leq r \leq \infty$, a $C^{r}$ local section $f$ of $E$, defined on an open subset $U$, induces a $C^{r-k}$ local section $j^{k} f: U \rightarrow E^{k}: x \mapsto j^{k} f(x)$ of $E^{k}$, called the $k$-jet extension of $f$.

Definition 1.1. A subset $\Omega$ of $E^{k}$ is called a differential relation of order $k$. It is said to be open if it is an open subset. A section of $E^{k}$ whose values are in $\Omega$ is called a section of $\Omega$. A $C^{k}$ section of $E$ whose $k$-jet extension is 
a section of $\Omega$ is called a solution of $\Omega$. A section of $E^{k}$ that coincides with the $k$-jet extension of some section of $E$ is said to be holonomic.

Observe that solutions of $\Omega$ and holonomic sections of $\Omega$ are in one to one correspondence.

Let $\Omega$ be a differential relation on the manifold $M$. We will be considering families of sections of $\Omega$ parameterized by cubes $S=[0,1]^{p}, p \geq 1$. Those are defined to be continuous maps $f: M \times S \rightarrow E^{k}$, such that for each $s$ in $S$, the partial map $f_{s}: M \rightarrow E^{k}$ (obtained by restricting the map $f$ to $M \times\{s\})$ is a section of $\Omega$. A homotopy of sections of $\Omega$ is a family parameterized by $S=[0,1]$. Concerning local sections of $\Omega$, we need to introduce some terminology. Let $\left(A, A^{\prime}\right)$ denote a nested pair of compact subsets of $M$. The word "nested" indicates that $A^{\prime} \subset A$.

- A family of sections of $\Omega$ defined near $A$ is a family defined on $U \times S$ for some neighborhood $U$ of $A$.

- Two families of sections $f_{s}$ and $g_{s}$ are said to coincide near $A$ if there exists a neighborhood $U$ of $A$ on which both $f_{s}$ and $g_{s}$ are defined, and for which $\left.f_{s}\right|_{U}=\left.g_{s}\right|_{U}$ for all $s$ in $S$.

- A family of sections $g_{s}$ defined near $A$ is said to extend another family $f_{s}$ defined near $A^{\prime}$ if $g_{s}$ and $f_{s}$ coincide near $A^{\prime}$.

- Two families of sections $f_{s}$ and $g_{s}$ defined near $A$ are said to be $h o$ motopic if there exists a third family $h_{s, t}$ defined near $A$ with $(s, t)$ in $S \times[0,1]$, such that $h_{s, 0}$ coincides with $f_{s}$ near $A$ and $h_{s, 1}$ coincides with $g_{s}$ near $A$.

- A homotopy $h_{s, t}$ is said to be stationary near $A$ for $s$ in $S^{\prime} \subset S$ if there exists a neighborhood $U$ of $A$ for which $h_{s, t}=h_{s, 0}$ on $U$, for $t$ in $[0,1]$, and for $s$ in $S^{\prime}$.

Similar definitions apply to sections of $E$ and to solutions of $\Omega$, with one restriction : families $f: M \times S \rightarrow E$ of $C^{k}$ sections of $E$ are required to be $C^{k}$-continuous, that is, their $k$-jet extension $j^{k} f: M \times S \rightarrow E^{k}$ is required to be a continuous map. To obtain solutions that are of smoothness class $C^{r}$, with $k \leq r \leq \infty$, one should everywhere consider $C^{r}$ sections of $E$ and $C^{k-r}$ sections of $E^{k}$ only. Also, when the relation considered is open, any (continuous) family of $C^{r}$ sections of $E$ can be approximated by a smooth family of $C^{r}$ sections, that is a smooth map $M \times S \rightarrow E$. 
In the sequel, $S^{\prime}$ will always denote a subset of $S=[0,1]^{p}$ consisting of the union of some of its faces.

Definition 1.2 (The parametric h-principle). The relation $\Omega \subset E^{k}$ is said to satisfy the parametric h-principle on $M$ (near a subset $A$ of $M$ ) if any family of sections of $\Omega$ (defined near $A$ ) is homotopic, among families of sections of $\Omega$ (defined near $A$ ), to a family of holonomic sections of $\Omega$. If the sections are already holonomic on $M$ (or near $A$ ) for $s$ in $S^{\prime}$, we may assume them to remain holonomic during the homotopy. (Equivalently, the homotopy may be assumed to be stationary for $\mathrm{s}$ in $S^{\prime}$ ).

Remark 1.3. Observe that if a differential relation $\Omega$ satisfies the parametric h-principle, then its solutions satisfy some kind of uniqueness property. Indeed, let $f_{0}$ and $f_{1}$ be two solutions of $\Omega$ whose $k$-jet extensions are homotopic among sections of $\Omega$. Let $f_{t}^{1}$ be such a homotopy. Then the family $f_{t}^{1}$ is homotopic to a family $f_{t}$ of holonomic sections of $\Omega$ via a homotopy that is stationary for $t \in\{0,1\}$. In particular, the two solutions $f_{0}$ and $f_{1}$ are homotopic among solutions of $\Omega$.

Remark 1.4. The relation $\Omega$ satisfies the parametric h-principle on $M$ if and only if the map $j^{k}: \operatorname{Sol}(\Omega) \rightarrow \Gamma(\Omega)$ is a weak homotopy equivalence, where $\operatorname{Sol}(\Omega)$ is the set of global solutions of $\Omega$ and where $\Gamma(\Omega)$ is the set of global sections of $\Omega$ (cf. [10] $(C)$ p. 16).

\subsection{Invariant relations.}

Definition 1.5. An isotopy of the manifold $M$ is a family $\varphi_{t}, t$ in $[0,1]$, of diffeomorphisms of $M$ such that the map $\varphi: M \times[0,1] \rightarrow M:(x, t) \mapsto$ $\varphi_{t}(x)$ is smooth and $\varphi_{0}=\operatorname{Id}_{M}$. Consider a foliation $\mathcal{F}$ on $M$. A foliated isotopy of $(M, \mathcal{F})$ is an isotopy $\varphi_{t}$ of $M$ that preserves the foliation $\mathcal{F}$, that is, $\left(\varphi_{t}\right)_{*}(T \mathcal{F})=T \mathcal{F}$ for all $t$ in $[0,1]$. Finally, two sets $A$ and $A^{\prime}$, with $A^{\prime} \subset A$ are said to be isotopic (respectively foliated isotopic) if for every neighborhood $U$ of $A^{\prime}$, there exists an isotopy (respectively a foliated isotopy) $\varphi_{t}^{U}$ of $M$ such that

- $\varphi_{t}^{U}$ is stationary near $A^{\prime}$,

- $\varphi_{t}^{U}(A) \subset A$ for all $t$,

- $\varphi_{1}^{U}(A) \subset U$. 
Definition 1.6. Let $\pi: E \rightarrow M$ be a locally trivial fibration. An isotopy $\varphi_{t}$ of $M$ is said to operate on local sections of $E$ if we are given an isotopy $\bar{\varphi}_{t}$ of $E$ covering $\varphi_{t}$ (i.e. $\pi \circ \bar{\varphi}_{t}=\varphi_{t} \circ \pi$ ) satisfying the following property:

if $\varphi_{t}$ coincides with $\varphi_{t_{0}}$ on an open subset $U$ for all $t \geq t_{0}$, then $\bar{\varphi}_{t}$ coincides with $\bar{\varphi}_{t_{0}}$ on $\pi^{-1}(U)$ for all $t \geq t_{0}$.

In this situation, if $f:\left.U \rightarrow E\right|_{U}$ is a local section of $E$ defined on some open subset $U$ of $M$, then $\varphi_{t} \cdot f=\bar{\varphi}_{t}^{-1} \circ f \circ \varphi_{t}$ is a local section of $E$ defined on $\varphi_{t}^{-1}(U)$. This operation on local sections induces an operation on $E^{k}$ as follows : $\varphi_{t} \cdot\left(j^{k} f(x)\right)=j^{k}\left(\varphi_{t} \cdot f\right)\left(\varphi_{t}^{-1}(x)\right)$.

Definition 1.7 (Invariant relations). The differential relation $\Omega \subset E^{k}$ is said to be invariant under the isotopy $\varphi_{t}$ if $\varphi_{t}$ operates on local sections of $E$, and if $\Omega$ is invariant under the induced operation on $E^{k}$ (i.e. $\varphi_{t} \cdot \Omega=\Omega$ ). The relation $\Omega$ is said to be invariant (respectively foliated invariant) if it is invariant under all isotopies of $M$ (respectively all foliated isotopies of $(M, \mathcal{F}))$.

\subsection{Local h-principle, h-principle for extensions.}

Definition 1.8 (The local parametric h-principle). A differential relation is said to satisfy the local parametric h-principle if it satisfies the parametric h-principle near any point.

Proposition 1.9 ([10] $B_{2}$, p. 37). Any open differential relation satisfies the local parametric h-principle.

Definition 1.10 (The parametric h-principle for extensions). Let $\left(A, A^{\prime}\right)$ be a nested pair of compact subsets of $M$. A differential relation $\Omega$ defined on $M$ is said to satisfy the parametric h-principle for extensions of solutions form $A^{\prime}$ to $A$, or on the pair $\left(A, A^{\prime}\right)$, if any family $f_{s}$ of sections of $\Omega$ defined near $A$ and holonomic near $A^{\prime}$ is homotopic to a family of holonomic sections, through a homotopy that is stationary near $A^{\prime}$. Moreover, if the sections $f_{s}$ are already holonomic near $A$ for $s$ in $S^{\prime}$, then the homotopy may be chosen to be stationary near $A$ for $s$ in $S^{\prime}$.

Lemma 1.11 ([10]). Let $\Omega$ be a differential relation on the manifold $M$, and let $\left(A, A^{\prime}\right)$ be a nested pair of isotopic compact subsets of $M$. If $\Omega$ is invariant under all isotopies $\varphi_{t}^{U}$, where $U$ runs through the set of neighborhoods of $A^{\prime}$, then the parametric h-principle for extensions holds on the pair $\left(A, A^{\prime}\right)$. 
Proposition $1.12\left([\mathbf{1 0}]\left(A^{\prime}\right)\right.$, p. 40). Let $\Omega$ be a differential relation on $M$, and let $M=\cup_{i>0} K_{i}$ be an exhaustion of $M$ by compact subsets (i.e., $K_{i} \subset K_{i+1}$ and $\left.M=\cup_{i \geq 0} K_{i}\right)$. Suppose that $\Omega$ satisfies the parametric $h$ principle on $K_{0}$, as well as the parametric h-principle for extensions on all pairs $\left(K_{i+1}, K_{i}\right)$. Then $\Omega$ satisfies the parametric h-principle on $M$.

Consider an open differential relation $\Omega$ on a manifold $M$ endowed with a foliation $\mathcal{F}$.

Theorem $1.13\left([10]\left(B_{1}\right)\right.$, p. 41; $\left(C_{1}\right)$, p. 42; $\left(C_{3}^{\prime}\right)$, p. 43). Let $\left(A, A^{\prime}\right)$ be a nested pair of compact subsets of $M$ such that the compact $C=\overline{A-A^{\prime}}$ is contained in an embedded submanifold $M_{0}$ of $M$, of codimension at least one. If the relation $\Omega$ is invariant, then it satisfies the parametric h-principle for extensions on $\left(A, A^{\prime}\right)$. If the relation $\Omega$ is only foliated invariant, but the submanifold $M_{0}$ intersects the foliation $\mathcal{F}$ transversely, then $\Omega$ satisfies the parametric h-principle for extensions on $\left(A, A^{\prime}\right)$ as well.

Remark 1.14. The hypothesis of the previous theorem can be weakened (without affecting the conclusion) as follows : $C$ consists of a finite union of compact sets, each contained in an embedded submanifold of codimension at least one (transverse to $\mathcal{F}$ ) (cf. [10] $\left(A^{\prime}\right)$, p. 40).

\section{Outline of the proof.}

We begin this section with a rough outline of the proof of Theorem 0.1. Let $\Omega \subset E^{k}$ be an open, invariant differential relation on a manifold $M$. Fixing a section $\beta$ of $\Omega$, the procedure to deform $\beta$ into a holonomic section of $\Omega$ is sketched below (the case of a family of sections is treated similarly). The hypotheses on $\Omega$ imply the following two facts.

(1) Since the relation $\Omega$ is open, it satisfies the local h-principle (cf. Proposition 1.9). Thus, for any given point $x$ in $M$, the section $\beta$ can be deformed into a holonomic sections on a sufficiently small neighborhood of $x$.

(2) Since $\Omega$ is invariant, it satisfies the h-principle for extensions on any pair $\left(A, A^{\prime}\right)$ of isotopic compact subsets (cf. Lemma 1.11). Thus, if $\beta$ can be deformed into a holonomic section near $A^{\prime}$, it can also be deformed into a holonomic section near $A$.

Starting from a holonomic section $\omega$ of $\Omega$ defined on a neighborhood $U$ of a point $x$ and homotopic to $\left.\beta\right|_{U}$, one tries to extend $\omega$ as far as possible. 
It can certainly be extended to any "large" open ball containing $x$ (cf. (2)). But when trying to go further, one has to understand how to deal with the topology of $M$. A good grasp on the latter is provided by a proper, positive Morse function on $M$, that is, a proper map $f: M \rightarrow[0, \infty)$ whose singular points are nondegenerate and lie on distinct levels. The term proper indicates that for any pair $a<b$ of real numbers, the set $f^{-1}([a, b])$ is compact.

Let $a<b$ be two noncritical values of $f$, and let $\omega$ be a holonomic section of $\Omega$, defined on a neighborhood of $f^{-1}([0, a])$ and homotopic to $\beta$. Provided $[a, b]$ does not contain a critical value of $f$, the set $f^{-1}([0, b])$ is isotopic to $f^{-1}([0, a])([17])$. The holonomic section $\omega$ can therefore be extended to a neighborhood of $f^{-1}([0, b])$ (cf. (2)). If $[a, b]$ contains a critical value of $f$ corresponding to a critical point $x$, the set $f^{-1}([0, b])$ is obtained from $f^{-1}([0, a])$ by gluing a closed disk $D^{k}$ along its boundary, in the sense that $f^{-1}([0, b])$ is isotopic to $f^{-1}([0, a]) \cup_{\partial D^{k}} D^{k}([17])$. The dimension $k$ of the disk coincides with the number of negative eigenvalues of the Hessian of $f$ at $x$. Thus, to extend $\omega$ through $x$, one needs to be able to extend a holonomic section of $\Omega$, homotopic to $\beta$, defined on a neighborhood of the boundary of an embedded disk $D^{k}$, to a holonomic section of $\Omega$, homotopic to $\beta$, defined on a neighborhood of the entire disk. (In other words, one needs the relation $\Omega$ to satisfy the h-principle for extensions on the pair $\left.\left(D^{k}, \partial D^{k}\right)\right)$. This can be done as long as $k<\operatorname{dim} M$ (cf. Theorem 1.13), and constitutes the key step of the proof of Theorem 0.1 .

The restriction $k<\operatorname{dim} M$ explains the dichotomy between closed and open manifolds. Indeed, if $M$ is open, we may assume that $f$ has no local maxima, or equivalently, that the disks we glue are never of maximal dimension. Beginning with a holonomic section $\omega$ of $\Omega$ defined near $f^{-1}(0)$ and homotopic to $\beta$, we can therefore extend it to $f^{-1}([0, b])$ for larger and larger values of $b$, eventually obtaining a global holonomic section of $\Omega$ (cf. Proposition 1.12).

Introducing the gradient $\nabla f$ of $f$ with respect to some Riemannian metric, one observes that its flow yields an isotopy between $f^{-1}([0, b])$ and $f^{-1}([0, a]) \cup_{\partial D^{k}} D^{k}$, where $D^{k}$ is now the set of points in $f^{-1}([a, b])$ lying on trajectories $\theta(t)$ "converging" to $x$, in the sense that $\lim _{t \rightarrow+\infty} \theta(t)=x$. It is useful to adopt this point of view when dealing with foliated manifolds.

Consider now the corresponding problem in the foliated case. Let $\Omega$ be an open, foliated invariant differential relation defined on a uniformely open foliated manifold $(M, \mathcal{F})$. Let $f: M \rightarrow[0, \infty)$ satisfy the hypotheses $a), b$ ) and $c$ ) of Definition 0.3. We may take advantage of what is already known. In particular, the facts (1) and (2) are still true here, provided we 
restrict ourselves to foliated isotopies. The key step is also valid in this context, provided the embedded disk $D^{k}$, does not only have codimension at least one, but intersects $\mathcal{F}$ transversely as well (cf. Theorem 1.13). The extra difficulties one faces come from the restriction to foliated isotopies. Indeed, up to foliated isotopies, the passage from $f^{-1}([0, a])$ to $f^{-1}([0, b])$ does not correspond anymore to gluing some disk (nor even some family of disks). It corresponds instead to gluing the "skeleton" consisting of the set of bounded trajectories of the leafwise gradient vector field of $f$ associated to some Riemannian metric $g$ (cf. Definition 4.1 and Lemma 4.5). The skeleton can be very complicated due to two phenomena:

i) the leafwise critical points of $f$ (Definition 3.2) can be leafwise degenerate (Remark 3.5),

ii) the leafwise critical points of $f$ are not isolated in $M$ (Remark 3.3).

Problem i) makes it hard to describe the topological structure of the skeleton already locally, near the foliated singular locus $\Sigma_{f}$ (Definition 3.2). Fortunately, to apply the key step, we do not need to know the exact topological type of the skeleton, it suffices to know that it is contained in a finite union of compact subsets of submanifolds of codimension at least one, intersecting $\mathcal{F}$ transversely (cf. Remark 1.14). When the latter holds in a neighborhood of $\Sigma_{f}$, the metric $g$ is said to be nice. The construction of a nice metric is detailed in Section 5. As it requires the foliated singular locus of $f$ to be stratified according to Thom-Boardman (Subsection 3.1), the function $f$ will be assumed to be strongly $\mathcal{F}$-generic (Definition 3.23). As noticed in Remark 3.24, this extra assumption is not restrictive. The construction of the metric is done by successive extensions from a neighborhood of one stratum to the next (with respect to some natural order on the set of strata).

Problem ii), on the other hand, makes it hard to have a grasp on the global structure of the skeleton due to the presence of trajectories $\theta(t)$ for which both $\lim _{t \rightarrow+\infty} \theta(t)$ and $\lim _{t \rightarrow-\infty} \theta(t)$ are in $f^{-1}([a, b])$. For such a trajectory, the structure of the skeleton near $\lim _{t \rightarrow-\infty} \theta(t)$ is quite complicated, more so that the stable and unstable manifolds of distinct critical points may not be assumed to intersect transversely. Fortunately, this difficulty vanishes if we approximate $f$ by a function whose leafwise critical points are isolated with respect to the leaf topology (cf. Proposition 3.21), as it allows one to "slice" $M$ sufficiently finely to (more or less) avoid having trajectories $\theta(t)$ for which both $\lim _{t \rightarrow+\infty} \theta(t)$ and $\lim _{t \rightarrow-\infty} \theta(t)$ lie in the same slice (cf. Section 6). 


\section{Genericity.}

This section is organized as follows. The first subsection shows how to adapt Boardman's construction of a natural stratification of the singular locus of a smooth map ([3]) to the foliated case. We use Mather's description of the Thom-Boardman stratification [14]. The second subsection shows that generically, the leafwise critical points of a real-valued function are isolated with respect to the leaf topology. This is a consequence of a result mentioned in [13] (6.1 p. 29) and proved along a scheme that appears in [12]. The third subsection defines (strongly) $\mathcal{F}$-generic functions and exhibits some of their properties. We begin with recalling the statement of Thom's transversality theorem which plays a crucial role throughout this section, defining the terms (nondegenerate) leafwise critical point and foliated singular locus used in the previous section, and briefly recalling from [6] the codimension one case.

Given two manifolds $M$ and $N$, the set $C^{\infty}(M, N)$ is endowed hereafter with the fine (or Whitney) $C^{\infty}$ topology. A subset of a topological space is residual if it is a countable intersection of dense open subsets. It is a classical result (e.g. [8]) that a residual subset of $C^{\infty}(M, N)$ is dense. Notice that a countable intersection of residual sets is still residual (while an intersection of dense sets is not dense in general). A condition on smooth functions $f$ in $C^{\infty}(M, N)$ is said to be generic if it is satisfied by all functions in a residual subset of $C^{\infty}(M, N)$. Recall that a smooth map $f: M \rightarrow P$ intersects an embedded submanifold $W$ of $P$ transversely at a point $x$ in $M$ if and only if either $f(x) \notin W$, or $f(x) \in W$ and

$$
f_{*_{x}} T_{x} M+T_{f(x)} W=T_{f(x)} P,
$$

and that $f$ intersects $W$ transversely if (1) holds for all $x$ in $f^{-1}(W)$. In that situation, the set $f^{-1}(W)$ is an embedded submanifold of $M$. If the map $f$ is the inclusion of a submanifold $W^{\prime}$, we say that the submanifolds $W$ and $W^{\prime}$ intersect transversely. Finally, the set of $k$-jets of local maps $U \subset M \rightarrow N$ is denoted by $J^{k}(M, N)$.

Theorem 3.1 (Thom Transversality Theorem). Let $W$ be an embedded submanifold of $J^{k}(M, N)$. The set of smooth maps $f$ whose $k$-jet extension

$$
j^{k} f: M \rightarrow J^{k}(M, N)
$$

is transverse to $W$ is residual in $C^{\infty}(M, N)$. 
A proof of the previous theorem can be found in [8].

Definition 3.2. Let $(M, \mathcal{F})$ be a foliated manifold, and let $f: M \rightarrow \mathbb{R}$ be a smooth function.

- A point $x$ at which $d\left(\left.f\right|_{F_{x}}\right)$ vanishes is called a leafwise critical point of $f$.

- A point $x$ at which $d\left(\left.f\right|_{F_{x}}\right)$ vanishes and $d^{2}\left(\left.f\right|_{F_{x}}\right): T_{x} \mathcal{F} \times T_{x} \mathcal{F} \rightarrow \mathbb{R}$ has maximal rank is called a nondegenerate leafwise critical point of $f$.

- The foliated singular locus of $f$ (with respect to the foliation $\mathcal{F}$ ) is the set of leafwise critical points of $f$, it is denoted by $\Sigma_{f}$.

\section{Remarks 3.3.}

- An ordinary critical point of $f$ is of course a leafwise critical point as well, but leafwise critical points persist in nearby leaves, so that they typically come in $q$-parameter families, where $q$ is the codimension of $\mathcal{F}$ (cf. the codimension one case below).

- An ordinary critical point of $f$ can very well be nondegenerate as ordinary critical point and degenerate as leafwise critical point. A critical point of $f$ that is nondegenerate as leafwise critical point is called a leafwise nondegenerate critical point of $f$.

Example 3.4. Ferry and Wasserman describe in [6] normal forms for generic real-valued functions on a manifold endowed with a codimension one foliation. Part of their findings is that generically $\Sigma_{f}$ is a closed onedimensional submanifold that intersects the foliation $\mathcal{F}$ transversely except along a discrete set of points. Transverse intersection points are nondegenerate leafwise critical points, while tangency points are degenerate leafwise critical points of birth type, a typical example of which is the point $(0,0)$ for the map

$$
\mathbb{R}^{2} \rightarrow \mathbb{R}:(x, y) \mapsto x^{3}-3 x y,
$$

defined on the manifold $\mathbb{R}^{2}$ endowed with the foliation by lines $\mathbb{R} \times\{y\}$.

Remark 3.5. A foliated manifold does not generally admit functions with no degenerate leafwise critical points. Indeed, for codimension one foliations, the foliated singular locus of such a function is a (not necessarily connected) closed transversal intersecting every compact leaf. The Reeb foliation on $S^{3}$, for example (cf. [4] p. 93), has no closed transversals intersecting the torus leaf (cf. [4] p. 147). 


\subsection{The Thom-Boardman stratification.}

Let $\mathcal{E}_{n}$ denote the set of germs of smooth maps $\mathbb{R}^{n} \rightarrow \mathbb{R}$ at the origin. The set $\mathcal{E}_{n}$ is a local ring, whose maximal ideal, denoted by $\mathfrak{m}_{n}$, is the set of germs vanishing at 0 . Given an ideal $\mathcal{I}$ of the $\operatorname{ring} \mathcal{E}_{n}$, we use the notation $\mathcal{I}^{k}$ for the product $\mathcal{I} \cdot \ldots \cdot \mathcal{I}$ of $k$ copies of $\mathcal{I}$. Finally, the symbol $D_{i}$ stands for the derivation $\frac{\partial}{\partial x_{i}}$, where $x_{1}, \ldots, x_{n}$ are the standard coordinates on $\mathbb{R}^{n}$.

Definition 3.6. Let $\mathcal{I}$ be a finitely generated ideal of $\mathcal{E}_{n}$.

i) The rank of $\mathcal{I}$, denoted by $\operatorname{rk}(\mathcal{I})$, is the dimension of $\left(\mathcal{I}+\mathfrak{m}_{n}^{2}\right) / \mathfrak{m}_{n}^{2}$ as real vector space.

ii) The ideal generated by $\mathcal{I}$ and the set $\Gamma_{r}(\mathcal{I})$ of $r \times r$ minors of the Jacobian matrix $\left(D_{i} f_{j}\right)_{1 \leq i \leq n, 1 \leq j \leq a}$, where $f_{1}, \ldots, f_{a}$ is a set of generators for $\mathcal{I}$, is denoted by $\Delta_{r}(\mathcal{I})$ and called a Jacobian extension of $\mathcal{I}$. Notice that the ideal $\Delta_{r}(\mathcal{I})$ (unlike the set $\Gamma_{r}(\mathcal{I})$ ) does not depend on the choice of generators for $\mathcal{I}$.

iii) The ideal $\Delta_{r+1}(\mathcal{I})$ with $r=r k(\mathcal{I})$ is denoted by $\delta(\mathcal{I})$. Notice that when $\mathcal{I}$ is proper, the ideal $\delta(\mathcal{I})$ is proper as well.

iv) The Boardman symbol of $\mathcal{I}$ is the infinite sequence $I(\mathcal{I})=\left(n-r_{1}, n-\right.$ $\left.r_{2}, \ldots, n-r_{\ell}, \ldots\right)$, where $r_{\ell}=\operatorname{rk}\left(\delta^{\ell-1}(\mathcal{I})\right)$.

Let $J^{k}(n, p)$ denote the set of $k$-jets of maps $\left(\mathbb{R}^{n}, 0\right) \rightarrow\left(\mathbb{R}^{p}, 0\right)$ at the origin. Given a jet $z=j^{k} f(0)$ in $J^{k}(n, p)$, represented by a map $f$, consider the ideal

$$
\mathcal{I}(z) \stackrel{\text { def. }}{=}(f)+\mathfrak{m}_{n}^{k+1},
$$

where $(f)$ denotes the ideal generated by the germs of the components $f_{1}, \ldots, f_{p}$ of $f$ at 0 . The Boardman symbol of the jet $z$, denoted by $I(z)$, is defined to be the Boardman symbol of the ideal $\mathcal{I}(z)$ truncated at order $k$ (notice that $I(\mathcal{I}(z))$ is of the type $\left.\left(i_{1}, \ldots, i_{k}, 0, \ldots, 0, \ldots\right)\right)$. Given a sequence $I$ of $k$ nonnegative integers, let $\Sigma^{I} \subset J^{k}(n, p)$ denote the set of $k$-jets whose Boardman symbol is $I$.

Proposition $3.7([3])$. Let $I=\left(i_{1}, \ldots, i_{k}\right)$. The set $\Sigma^{I}$ is nonempty if and only if $n \geq i_{1} \geq i_{2} \geq \ldots \geq i_{k}$, and either $i_{1}>n-p$, or $i_{1}=n-p$ and $i_{1}=i_{2}=\ldots=i_{k}$. Moreover $\Sigma^{I}$ is an embedded submanifold of $J^{k}(n, p)$ whose codimension is given by the formula

$$
\operatorname{cod} \Sigma^{I}=\left(p-n+i_{1}\right) \mu_{I}-\left(i_{1}-i_{2}\right) \mu_{s I}-\ldots-\left(i_{k-1}-i_{k}\right) \mu_{s^{k-1} I},
$$


where $s^{j} I$ denotes the sequence $\left(i_{j+1}, \ldots, i_{k}\right)$, and where $\mu_{\left(i_{1}, \ldots, i_{\ell}\right)}$ is the number of sequences $\left(j_{1}, j_{2}, \ldots, j_{\ell}\right)$ of integers satisfying

- $j_{1} \geq j_{2} \geq \ldots \geq j_{\ell}$

- $i_{r} \geq j_{r} \geq 0$ for all $r$, and $j_{1}>0$.

Given two manifolds $N$ and $P$, of respective dimensions $n$ and $p$, let $J^{k}(N, P)$ denote the manifold of $k$-jets of local functions on $N$ with values in $P$. The manifold $J^{k}(N, P)$ is a bundle over $N \times P$ whose fibers are diffeomorphic to $J^{k}(n, p)$, and whose structure group is the product of the group of invertible jets in $J^{k}(n, n)$ with the group of invertible jets in $J^{k}(p, p)$. For any sequence $I=\left(i_{1}, \ldots, i_{k}\right)$ of nonnegative integers, let $\Sigma^{I} \subset J^{k}(N, P)$ denote the subset

$$
\Sigma^{I}=\bigcup_{(x, y) \in N \times P} \Sigma_{x, y}^{I}
$$

where $\Sigma_{x, y}^{I}$ corresponds to $\Sigma^{I}$ under the identification of the fiber of $J^{k}(N, P)$ at $(x, y)$ with the manifold $J^{k}(n, p)$. Since $\Sigma^{I} \subset J^{k}(n, p)$ is invariant under the action of the structure group of the bundle $\pi: J^{k}(N, P) \rightarrow N \times P$, the set $\Sigma^{I} \subset J^{k}(N, P)$ is well-defined. Clearly $\Sigma^{I}$ is an embedded submanifold of $J^{k}(N, P)$ whose codimension is given by formula (2). Given a smooth function $f: N \rightarrow P$ whose $k$-jet extension intersects $\Sigma^{I}$ nontrivially and transversely at $x_{o}$, let

$$
\Sigma_{f}^{I} \stackrel{\text { def. }}{=} j^{k} f^{-1}\left(\Sigma^{I}\right)
$$

$\Sigma_{f}^{I}$ is a submanifold of $N$ in a neighborhood of $x_{o}$, and the following holds.

Proposition 3.8 ([3], Theorem (6.2)).

$$
\operatorname{dim} \operatorname{Ker} d\left(\left.f\right|_{\left.\Sigma_{f}^{\left(i_{1}, \ldots, i_{k}\right)}\right)}\right)\left(x_{o}\right)=\ell \quad \Longleftrightarrow \quad j^{k+1} f\left(x_{o}\right) \in \Sigma^{\left(i_{1}, \ldots, i_{k}, \ell\right)} .
$$

Let $M$ be an $m$-dimensional manifold endowed with a foliation $\mathcal{F}$ of dimension $n$ and codimension $q$. The inclusion of a leaf $F$ into $M$ gives rise to a submersion

$$
r_{F}: \pi^{-1}(F \times \mathbb{R}) \subset J^{k}(M, \mathbb{R}) \rightarrow J^{k}(F, \mathbb{R}): j^{k} f(x) \mapsto j^{k}\left(\left.f\right|_{F}\right)(x) .
$$

For every sequence $I=\left(n=i_{1}, \ldots, i_{k}\right)$ of integers, let

$$
\Sigma_{\mathcal{F}}^{I} \stackrel{\text { def. }}{=} \bigcup_{F \in\{\text { leaves of } \mathcal{F}\}} r_{F}^{-1}\left(\Sigma^{I}\right)
$$


The set $\Sigma_{\mathcal{F}}^{I}$ is an embedded submanifold of $J^{k}(M, \mathbb{R})$ whose codimension coincides with that of $\Sigma^{I}$ in $J^{k}(n, 1)$. Indeed, given a foliated chart $(U, \varphi)$ centered at a point $x$, we have $\pi^{-1}(U \times \mathbb{R}) \cap \Sigma_{\mathcal{F}}^{I} \simeq U \times \mathbb{R} \times r^{-1}\left(\Sigma^{I}\right)$, where $r$ is the submersion $J^{k}(m, 1) \rightarrow J^{k}(n, 1): j^{k} f(0) \mapsto j^{k}\left(\left.f\right|_{\mathbb{R}^{n} \times\{0\}}\right)(0)$.

Definition 3.9. In $C^{\infty}(M)$ endowed with the fine $C^{\infty}$ topology, let $\mathcal{A}$ denote the set of functions whose $k$-jet extension is transverse to $\Sigma_{\mathcal{F}}^{I}$ for all $I=\left(i_{1}, \ldots, i_{k}\right)$ and all $k=1,2, \ldots$

By Thom's Transversality Theorem, this set is residual. Now given $f$ in $\mathcal{A}$ and any sequence $I$ of length $k$, the set $\Sigma_{\mathcal{F}, f}^{I}=\left(j^{k} f\right)^{-1}\left(\Sigma_{\mathcal{F}}^{I}\right)$ is an embedded submanifold whose codimension equals that of $\Sigma_{\mathcal{F}}^{I}$ in $J^{k}(M, \mathbb{R})$; it is called a Boardman stratum. There are only finitely many nonempty Boardman strata, provided we ignore sequences ending with more than one zero. The last assertion follows from the observation that if $i_{k+1}>0$, the codimension of $\Sigma^{\left(I, i_{k+1}\right)}$ in $J^{k+1}(n, p)$ is strictly larger than that of $\Sigma^{I}$ in $J^{k}(n, p)$ (cf. [3] p. 47).

The following result is a consequence of Proposition 3.8.

Proposition 3.10. Let $f$ be an element of $\mathcal{A}$ and let $x_{o}$ be a point in $\Sigma_{\mathcal{F}, f}^{\left(i_{1}, \ldots, i_{k}\right)}$. Then

$$
\operatorname{dim}\left(T_{x_{o}} \mathcal{F} \cap T_{x_{o}} \Sigma_{\mathcal{F}, f}^{\left(i_{1}, \ldots, i_{k}\right)}\right)=\ell \quad \Longleftrightarrow \quad j^{k+1} f\left(x_{o}\right) \in \Sigma_{\mathcal{F}}^{\left(i_{1}, \ldots, i_{k}, \ell\right)}
$$

Proof. Since the problem is local, we may assume that $M$ is diffeomorphic to a product $F \times V$, with $F=\mathbb{R}^{n}, V=\mathbb{R}^{q}$, and where $\mathcal{F}$ corresponds to the "horizontal" foliation of $F \times V$ (whose leaves are the $F \times\{v\}$ 's). Let $u_{1}, \ldots, u_{n}$ and $v_{1}, \ldots, v_{q}$ denote the standard coordinates in $F$ and $V$ respectively. To the smooth function $f: M \rightarrow \mathbb{R}$, we associate a smooth map $\tilde{f}: M \rightarrow \mathbb{R} \times V$ by setting $\tilde{f}(x)=(f(x), p(x))$, where $p$ denotes the natural projection $F \times V \rightarrow V$. The proof of Proposition 3.10 relies on the following three statements. Let $x$ be any point in $M$, and let $k$ be any positive integer, then

a) $j^{k} f(x) \in \Sigma_{\mathcal{F}}^{I} \subset J^{k}(M, \mathbb{R})$ if and only if $j^{k} \tilde{f}(x) \in \Sigma^{I} \subset J^{k}(M, \mathbb{R} \times V)$;

b) $j^{k} f$ intersects $\Sigma_{\mathcal{F}}^{I}$ transversely at $x$ if and only if $j^{k} \tilde{f}$ intersects $\Sigma^{I}$ transversely at $x$;

c) if $x$ belongs to $\Sigma_{\mathcal{F}, f}^{I}$, then $T_{x} \mathcal{F} \cap T_{x} \Sigma_{\mathcal{F}, f}^{I}=\operatorname{Ker} d\left(\left.\tilde{f}\right|_{\Sigma_{\mathcal{F}, f}^{I}}\right)(x)$. 
Assuming these statements proved, we conclude the proof of Proposition 3.10 as follows. Statements $a$ ) and $b$ ), combined with Proposition 3.8 imply that

$$
\operatorname{dim} \operatorname{Ker} d\left(\left.\tilde{f}\right|_{\Sigma_{\mathcal{F}, f}^{I}}\right)\left(x_{o}\right)=\ell \quad \Longleftrightarrow \quad j^{k+1} f\left(x_{o}\right) \in \Sigma_{\mathcal{F}}^{\left(i_{1}, \ldots, i_{k}, \ell\right)} .
$$

Statement $c$ ) implies that (5) is equivalent to (4).

Proof of a). Consider the map

$$
\begin{array}{rll}
J^{k}(M, \mathbb{R} \times V) & \stackrel{p_{1} \times p_{2}}{\longrightarrow} & J^{k}(M, \mathbb{R}) \times J^{k}(M, V) \\
j^{k} g(x) & \mapsto & \left(j^{k} g_{1}(x), j^{k} g_{2}(x)\right),
\end{array}
$$

where $g$ is a map $M \rightarrow \mathbb{R} \times V$, whose components are $g_{1}: M \rightarrow \mathbb{R}$ and $g_{2}: M \rightarrow V$. Let $\mathcal{P} \subset J^{k}(M, V)$ be the image of the jet extension $j^{k} p$, and let $\tilde{\mathcal{P}}=p_{2}^{-1}(\mathcal{P})$. It is sufficient to prove that in $J^{k}(M, \mathbb{R} \times V)$,

$$
\tilde{\mathcal{P}} \cap \Sigma^{I}=\tilde{\mathcal{P}} \cap p_{1}^{-1}\left(\Sigma_{\mathcal{F}}^{I}\right) .
$$

Let $z=\left(z_{1}, z_{2}\right)=\left(j^{k} f(x), j^{k} p(x)\right)=j^{k} \tilde{f}(x)$ be an element in $\tilde{\mathcal{P}}$. We assume for convenience that $x=0$. Let $\tilde{z}_{1}$ denote $j^{k}\left(\left.f\right|_{F \times\{0\}}\right)(x)$. We will prove, by induction on $0 \leq \ell \leq k$, that in $\mathcal{E}_{m}$,

$$
\delta^{\ell}(\mathcal{I}(z))=(v)+\delta^{\ell}\left(\mathcal{I}\left(\tilde{z}_{1}\right)\right),
$$

where $(v)$ denotes the ideal generated by the germs of the functions $v_{1}, \ldots, v_{q}$, and where $\delta^{\ell}\left(\mathcal{I}\left(\tilde{z}_{1}\right)\right)$ is thought of as being an ideal of $\mathcal{E}_{m}$. This will imply that $\operatorname{rk} \delta^{\ell}(\mathcal{I}(z))=q+\operatorname{rk} \delta^{\ell}\left(\mathcal{I}\left(\tilde{z}_{1}\right)\right)$ for all $0 \leq \ell \leq k$, hence that $z_{1}$ lies in $\Sigma_{\mathcal{F}}^{I}$ if and only if $z$ lies in $\Sigma^{I}$, which is the content of (6).

Observe that (7) clearly holds when $\ell=0$. Indeed, in a neighborhood of 0 in $M$, the function $f$ can be written as

$$
f(u, v)=f(u, 0)+\sum_{i=1}^{q} v_{i} h_{i}(u, v)
$$

for some smooth functions $h_{i}$. Then suppose that (7) holds for some $\ell \geq 0$. The ideal $\delta^{\ell+1}(\mathcal{I}(z))$ is generated by $\delta^{\ell}(\mathcal{I}(z))$ and the set $\Gamma_{r+1} \delta^{\ell}(\mathcal{I}(z))$ of $(r+1) \times(r+1)$ minors of the Jacobian matrix $\left(D_{i} f_{j}\right)_{1 \leq i \leq m, 1 \leq j \leq a}$, where $r=\operatorname{rk} \delta^{\ell}(\mathcal{I}(z))$, and where $f_{1}, \ldots, f_{a}$ is a set of generators for $\delta^{\ell}(\mathcal{I}(z))$. Since we may assume that $f_{1}=v_{1}, \ldots, f_{q}=v_{q}$ and that $f_{q+1}, \ldots, f_{a}$ are generators of $\delta^{\ell}\left(\mathcal{I}\left(\tilde{z}_{1}\right)\right)$, the set $\Gamma_{r+1} \delta^{\ell}(\mathcal{I}(z))$ is also the set of $(r-q+1) \times(r-q+1)$ minors 
of $\left(D_{i} f_{j}\right)_{1 \leq i \leq n, q+1 \leq j \leq a}$. Since the latter set coincides with $\Gamma_{r-q+1} \delta^{\ell}\left(\mathcal{I}\left(\tilde{z}_{1}\right)\right)$, we have

$$
\delta^{\ell+1}(\mathcal{I}(z))=(v)+\delta^{\ell}\left(\mathcal{I}\left(\tilde{z}_{1}\right)\right)+\Gamma_{r-q+1} \delta^{\ell}\left(\mathcal{I}\left(\tilde{z}_{1}\right)\right)=(v)+\delta^{\ell+1}\left(\mathcal{I}\left(\tilde{z}_{1}\right)\right) .
$$

Proof of b). Suppose that $j^{k} f(x) \in \Sigma_{\mathcal{F}}^{I}$. Observe that the identity (6) implies that $j^{k} f$ intersects $\Sigma_{\mathcal{F}}^{I}$ transversely at $x$ if and only if

$$
\begin{aligned}
T_{z} \tilde{\mathcal{P}} & =T_{z} \tilde{\mathcal{P}} \cap T_{z}\left(p_{1}^{-1} \Sigma_{\mathcal{F}}^{I}\right)+\left(j^{k} \tilde{f}\right)_{*_{x}}\left(T_{x} M\right) \\
& =T_{z}\left(\tilde{\mathcal{P}} \cap p_{1}^{-1} \Sigma_{\mathcal{F}}^{I}\right)+\left(j^{k} \tilde{f}\right)_{*_{x}}\left(T_{x} M\right) \\
& =T_{z}\left(\tilde{\mathcal{P}} \cap \Sigma^{I}\right)+\left(j^{k} \tilde{f}\right)_{*_{x}}\left(T_{x} M\right),
\end{aligned}
$$

where $z=j^{k} \tilde{f}(x)=\left(j^{k} f(x), j^{k} p(x)\right)=\left(z_{1}, z_{2}\right)$. So, in order to prove $\left.b\right)$, we need to show that $\Sigma^{I}$ and $\tilde{\mathcal{P}}$ intersect transversely at $z$, or equivalently that

$$
T_{z_{2}} \mathcal{P}+\left(p_{2}\right)_{*_{z}}\left(T_{z} \Sigma^{I}\right)=T_{z_{2}} J^{k}(M, V) .
$$

Since $J^{k}(M, V) \simeq M \times V \times J^{k}(m, q)$, and since $\mathcal{P}$ is transverse to $\{x\} \times V \times$ $J^{k}(m, q)$, it is sufficient to prove that $T_{p(x)} V$ and $T_{z_{2}} J^{k}(m, q)$ are contained in $\left(p_{2}\right)_{*_{z}}\left(T_{z} \Sigma^{I}\right)$.

Observe that if $\varphi_{X}^{t}$ denotes the local flow of a vector field $X$ defined on $M$, vanishing at $x$, then $j^{k}\left(\tilde{f} \circ \varphi_{X}^{t}\right)(x)$ belongs to $\Sigma^{I}$ for all $t$. Hence

$$
\xi_{X} \stackrel{\text { def. }}{=} \frac{d\left(j^{k}\left(\tilde{f} \circ \varphi_{X}^{t}\right)(x)\right)}{d t}(0) \in T_{z} \Sigma^{I} .
$$

Moreover, the set of vectors $\left(p_{2}\right)_{*_{z}}\left(\xi_{X}\right)$, as $X$ varies among vector fields vanishing at $x$, coincides with $T_{z_{2}} J^{k}(m, q)$. So it remains to prove that $T_{p(x)} V$ is contained in $\left(p_{2}\right)_{*_{z}}\left(T_{z} \Sigma^{I}\right)$ as well. This is easily seen once we notice that $T_{z} \Sigma^{I}$ contains $T_{\tilde{f}(x)}(\mathbb{R} \times V)$, and hence that $\left(p_{2}\right)_{*_{z}}\left(T_{z} \Sigma^{I}\right)$ contains $\left(p_{2}\right)_{*_{z}}\left(T_{\tilde{f}(x)}(\mathbb{R} \times V)\right)=T_{p(x)} V$.

Proof of $c$ ). From $a$ ) we know that $\Sigma_{\mathcal{F}, f}^{I}=\Sigma_{\tilde{f}}^{I}$. Besides, if $x$ lies in $\Sigma_{\mathcal{F}, f}^{I}$, then

$$
\begin{aligned}
\operatorname{Ker} d\left(\left.\tilde{f}\right|_{\Sigma_{\tilde{f}}^{I}}\right)(x) & =\operatorname{Ker} d \tilde{f}(x) \cap T_{x} \Sigma_{\tilde{f}}^{I} \\
& =T_{x} \mathcal{F} \cap T_{x} \Sigma_{\tilde{f}}^{I} .
\end{aligned}
$$


Let $f$ be an element of $\mathcal{A}$. We would like to interpret the first few $\Sigma_{\mathcal{F}, f}^{I}$ 's. Before doing so, we introduce the foliated second differential of $f$ (with respect to the foliation $\mathcal{F}$ ), that is, the map

$$
\bar{d}^{2} f: T_{\Sigma} \mathcal{F} \oplus T_{\Sigma} \mathcal{F} \rightarrow \mathbb{R}:(X, Y) \mapsto Y(\tilde{X}(f)),
$$

where $\tilde{X}$ is any local section of $T \mathcal{F}$ extending $X$. (It appeared already implicitely in Definition 3.2.) As in the nonfoliated case, it is easily checked that $\bar{d}^{2} f$ is well-defined and symmetric.

- $\Sigma_{\mathcal{F}, f}^{(n)}=\Sigma_{f}$ is the foliated singular locus of $f$ (according to Definition 3.2).

- $\Sigma_{\mathcal{F}, f}^{(n, 0)}$ is the set of nondegenerate leafwise critical points (still according to Definition 3.2).

- $\Sigma_{\mathcal{F}, f}^{(n, i)}$ is the set of leafwise critical points $x$ for which $\bar{d}^{2} f(x)$ has rank $n-i$.

- In general, as implied by Proposition $3.10, \Sigma_{\mathcal{F}, f}^{\left(i_{1}, \ldots, i_{k}\right)}$ is the set of points $x$ in $\Sigma_{\mathcal{F}, f}^{\left(i_{1}, \ldots, i_{k-1}\right)}$ where $\operatorname{dim}\left(T_{x} \mathcal{F} \cap T_{x} \Sigma_{\mathcal{F}, f}^{\left(i_{1}, \ldots, i_{k-1}\right)}\right)=i_{k}$.

Observe that $M$ is partitioned into the embedded submanifolds $\Sigma_{\mathcal{F}, f}^{I}$, where $I=\left(i_{1}, \ldots, i_{k}, 0\right)$ and $i_{k} \neq 0$. They intersect $\mathcal{F}$ transversely, in the sense that $T_{x} \Sigma_{\mathcal{F}, f}^{I} \cap T_{x} \mathcal{F}=\{0\}$ for all $x$ in $\Sigma_{\mathcal{F}, f}^{I}$. Finally, the submanifold $\Sigma_{\mathcal{F}, f}^{I}$ is of course not closed in general. In fact,

$$
\overline{\Sigma_{\mathcal{F}, f}^{\left(i_{1}, \ldots, i_{k}\right)}} \subset \underset{\left(j_{1}, \ldots, j_{\ell}\right) \geq\left(i_{1}, \ldots, i_{k}\right)}{ } \Sigma_{\mathcal{F}, f}^{\left(j_{1}, \ldots, j_{\ell}\right)},
$$

where the symbol $\geq$ refers to the lexicographical order on the set of tuples of nonnegative integers.

\subsection{Isolatedness of leafwise critical points.}

We will need (cf. Observation 6.2) to approximate an $\mathcal{F}$-generic function by one whose leafwise critical points are isolated with respect to the leaf topology. The purpose of this subsection is to prove that the latter property is generic (cf. Proposition 3.21).

Recall that $\mathcal{E}_{n}$ and $\mathfrak{m}_{n}$ denote the ring of germs of smooth maps $\mathbb{R}^{n} \rightarrow$ $\mathbb{R}$ at the origin and its maximal ideal respectively. The set of $k$-jets of 
maps $\left(\mathbb{R}^{n}, 0\right) \rightarrow(\mathbb{R}, 0)$ at the origin is denoted by $J^{k}(n)$. For $\ell>k$, the natural map $J^{\ell}(n) \rightarrow J^{k}(n): j^{\ell} f(0) \mapsto j^{k} f(0)$ is denoted by $\pi_{k}^{\ell}$. As before, $x_{1}, \ldots, x_{n}$ are the standard coordinates on $\mathbb{R}^{n}$.

Definition 3.11. The Jacobian ideal of an element $f \in \mathfrak{m}_{n}^{2}$, denoted by $\mathcal{J}(f)$, is the ideal of $\mathcal{E}_{n}$ generated by the partial derivatives $\frac{\partial f}{\partial x_{1}}, \ldots, \frac{\partial f}{\partial x_{n}}$ of $f$. We say that $f$ has codimension $k$ if $\operatorname{cod}(\mathcal{J}(f))=\operatorname{dim}_{\mathbb{R}}\left(\mathfrak{m}_{n} / \mathcal{J}(f)\right)=k$.

We will think of a singular element $z=j^{k} f(0)$ of $J^{k}(n)$ (i.e., $\left.d f(0)=0\right)$ as a polynomial function on $\mathbb{R}^{n}$, and define its Jacobian ideal and codimension accordingly.

The following is a consequence of Nakayama's lemma. A proof can be found in [12] (Appendix A.2., Proposition 2.2.).

Lemma 3.12. If $f-f(0) \in \mathfrak{m}_{n}^{2}$ has finite codimension, then 0 is an isolated singularity of $f$.

Definition 3.13. Two germs $f$ and $g$ in $\mathcal{E}_{n}$ are said to be equivalent if there exists a germ $\phi$ of local diffeomorphism $\left(\mathbb{R}^{n}, 0\right) \rightarrow\left(\mathbb{R}^{n}, 0\right)$ of $\mathbb{R}^{n}$ at the origin for which $f=g \circ \phi$.

Observe that equivalent germs have same codimension.

Definition 3.14. A germ $f \in \mathcal{E}_{n}$ is $k$-determined if for any element $g$ in $\mathfrak{m}_{n}^{k+1}$, the germ $f+g$ is equivalent to $f$.

Theorem 3.15 ([15]). If the codimension of $f \in \mathcal{E}_{n}$ is $k$, then $f$ is $(k+2)$ determined.

Consider in $J^{k}(n)$ the set $Z^{k}$ consisting of singular $k$-jets having codimension strictly larger than $k-2$.

Proposition 3.16. The set $Z^{k}$ is a real algebraic subset of the Euclidean space $J^{k}(n)$.

Before proving Proposition 3.16, let us mention some facts about real algebraic sets. Recall that a real algebraic subset of a Euclidean space $\mathbb{R}^{p}$ is the zero locus of a collection of polynomial functions defined on $\mathbb{R}^{p}$. Such a set is a variety when it is irreducible, that is, when it cannot be decomposed into the union of two proper real algebraic sets. A real algebraic subset $S$ of $\mathbb{R}^{p}$ can be written uniquely (up to reordering the factors) as a finite union

$$
S=V_{1} \cup \ldots \cup V_{n}
$$


of varieties, where no factor $V_{i}$ is contained in a factor $V_{j}$ with $i \neq j$. Such a decomposition is said to be minimal. Real algebraic sets can also be decomposed into smooth submanifolds :

Theorem 3.17 ([21]). Any real algebraic subset of $\mathbb{R}^{p}$ can be decomposed into a finite union of disjoint embedded submanifolds of $\mathbb{R}^{p}$.

The dimension of $S$ is defined to be the maximum dimension of the factors. The following result will be crucial later on (cf. proof of Proposition 3.20).

Theorem 3.18 ([21]). A proper subvariety of a variety has strictly smaller dimension.

Proof of Proposition 3.16. This type of argument is quite standard (see [12]). Let $f \in J^{k}(n)$ be a singular jet. First observe that $\operatorname{cod}(\mathcal{J}(f))>k-2$ if and only if $\operatorname{cod}\left(\mathcal{J}(f)+\mathfrak{m}_{n}^{k}\right)>k-2$. Indeed, suppose that $\operatorname{cod}\left(\mathcal{J}(f)+\mathfrak{m}_{n}^{k}\right) \leq k-2$. Then, in the following nested sequence of spaces

$$
\mathfrak{m}_{n} \supset \mathcal{J}(f)+\mathfrak{m}_{n}^{2} \supset \mathcal{J}(f)+\mathfrak{m}_{n}^{3} \supset \ldots \supset \mathcal{J}(f)+\mathfrak{m}_{n}^{k}
$$

equality must occur somewhere, that is, $\mathcal{J}(f)+\mathfrak{m}_{n}^{r}=\mathcal{J}(f)+\mathfrak{m}_{n}^{r+1}$ for some $r \leq k-1$. This implies that $\mathfrak{m}_{n}^{r} \subset \mathcal{J}(f)+\mathfrak{m}_{n}^{r+1}$. Hence $\mathfrak{m}_{n}^{k} \subset \mathfrak{m}_{n}^{r} \subset \mathcal{J}(f)$ (cf. [13] Proposition 4. p. 3). Therefore $\operatorname{cod}(\mathcal{J}(f))=\operatorname{cod}\left(\mathcal{J}(f)+\mathfrak{m}_{n}^{k}\right) \leq$ $k-2$. The other implication is obvious.

Now $\operatorname{cod}\left(\mathcal{J}(f)+\mathfrak{m}_{n}^{k}\right)>k-2$ is equivalent to

$$
\operatorname{dim}\left(\mathcal{J}(f)+\mathfrak{m}_{n}^{k}\right) / \mathfrak{m}_{n}^{k}<\operatorname{dim}\left(\mathfrak{m}_{n} / \mathfrak{m}_{n}^{k}\right)-(k-2) \stackrel{\text { def. }}{=} N
$$

The vector space $\left(\mathcal{J}(f)+\mathfrak{m}_{n}^{k}\right) / \mathfrak{m}_{n}^{k}$ is generated by elements of the type $x^{\alpha} \frac{\partial f}{\partial x^{i}}$, where $\alpha$ is a multi-index with $|\alpha| \leq k-2$. Therefore, its dimension is less than $N$ when the rank of a certain matrix $M$, whose coefficients are linear functions of the coefficients of the polynomial function $f$, is bounded by $N-1$; or equivalently, when all minors of $M$ of size $N$ and larger vanish.

The combination of Theorem 3.16 and Theorem 3.17 implies that

$$
Z^{k}=M_{1} \coprod \ldots \coprod M_{r}
$$

where each $M_{i}$ is an embedded submanifold of $J^{k}(n)$. 
Proposition 3.19. For all $\ell>k$, the real algebraic set $Z^{\ell}$ is a subset of the real algebraic set $\left(\pi_{k}^{\ell}\right)^{-1}\left(Z^{k}\right)$.

Proof. Let $f$ be an $\ell$-jet whose truncation $\pi_{k}^{\ell}(f)$ does not belong to $Z^{k}$, that is, $\operatorname{cod}\left(\mathcal{J}\left(\pi_{k}^{\ell}(f)\right)\right) \leq k-2$. Theorem 3.15 implies that $f$ is equivalent to $\pi_{k}^{\ell}(f)$. Hence $\operatorname{cod}(\mathcal{J}(f)) \leq k-2$ as well. Thus $f$ lies outside $Z^{\ell}$.

Proposition 3.20. The codimension $c_{k}$ of $Z^{k}$ in $J^{k}(n)$ is an unbounded function of $k$.

Proof. Consider the homogeneous polynomial $r=x_{1}^{k+1}+\ldots+x_{n}^{k+1}$ of degree $k+1$. The Jacobian ideal of $r$ coincides with $\left(x_{1}^{k}, \ldots, x_{n}^{k}\right)$, and contains $\mathfrak{m}_{n}^{n(k-1)+1}$. Thus

$$
\operatorname{cod}(r) \leq \operatorname{cod}\left(\mathfrak{m}_{n}^{n(k-1)+1}\right) \stackrel{\text { def. }}{=} a_{k} .
$$

Let $p$ be any polynomial of degree $k$. The codimension of $p+r$ is bounded by the codimension of $r$ (cf. [12] A.2., Theorem 2.7). Thus cod $(p+r) \leq a_{k}$ for all $p \in J^{k}(n)$. Let $\ell=a_{k}+2$. We have just shown that for all $p \in Z^{k}$, the set $\left(\pi_{k}^{\ell}\right)^{-1}(p)$ is not entirely contained in $Z^{\ell}$.

Now, let $Z^{k}=V_{1}^{k} \cup \ldots \cup V_{n_{k}}^{k}$ be the minimal decomposition of $Z^{k}$ into varieties. Let $\left(\pi_{k}^{\ell}\right)^{-1}\left(V_{j}^{k}\right)$ be denoted by $W_{j}$. The real algebraic set $W_{j}$ is irreducible as well. Because $Z^{\ell}=V_{1}^{\ell} \cup \ldots \cup V_{n_{\ell}}^{\ell} \subset\left(\pi_{k}^{\ell}\right)^{-1}\left(Z^{k}\right)$, the set $V_{i}^{\ell}$ coincides with $\left(V_{i}^{\ell} \cap W_{1}\right) \cup \ldots \cup\left(V_{i}^{\ell} \cap W_{n_{k}}\right)$. Since $V_{i}^{\ell}$ is irreducible, there exists an index $j_{i}$ such that $V_{i}^{\ell}=V_{i}^{\ell} \cap W_{j_{i}}$. The previous paragraph implies that $V_{i}^{\ell}$ is a proper subset of $W_{j_{i}}$. Hence, the codimension of $V_{i}^{\ell}$ is strictly larger than that of $W_{j_{i}}$ (Theorem 3.18). Thus, the codimension of $Z^{\ell}$ in $J^{\ell}(n)$ is strictly larger than that of $\left(\pi_{k}^{\ell}\right)^{-1}\left(Z^{k}\right)$, that is $\operatorname{cod} Z^{\ell}>\operatorname{cod} Z^{k}$.

Proposition 3.21. Let $M$ be a manifold endowed with a foliation $\mathcal{F}$. For $f$ in a residual subset $\mathcal{B}$ of $C^{\infty}(M)$, and for any leaf $F$ of $\mathcal{F}$, the critical points of $\left.f\right|_{F}$ are isolated in $F$.

Proof. Given a leaf $F$ of the foliation $\mathcal{F}$, define $Z^{k}(F) \subset J^{k}(F, \mathbb{R})$ to be

$$
Z^{k}(F)=\bigcup_{(x, y) \in F \times \mathbb{R}} Z^{k}(F)_{(x, y)},
$$

where $Z^{k}(F)_{(x, y)}$ corresponds to $Z^{k}$ under the identification of the fiber at $(x, y)$ of the bundle $J^{k}(F, \mathbb{R}) \rightarrow F \times \mathbb{R}$ with $J^{k}(n)$. Then, define $Z^{k}(\mathcal{F}) \subset$ 
$J^{k}(M, \mathbb{R})$ to be:

$$
Z^{k}(\mathcal{F})=\bigcup_{F \in\{\text { leaves of } \mathcal{F}\}} r_{F}^{-1}\left(Z^{k}(F)\right)
$$

where $r_{F}$ is defined by the expression (3). Locally, the set $Z^{k}(\mathcal{F})$ is the union of finitely many embedded submanifolds of $J^{k}(M, \mathbb{R})$ having codimension at least $c_{k}$. Hence, Thom's Transversality Theorem implies that, provided $k$ is large enough for $c_{k}>\operatorname{dim} M$, the set $\mathcal{B}$ of smooth real-valued functions on $M$ whose $k$-jet does not meet $Z^{k}(\mathcal{F})$ is residual in $C^{\infty}(M)$ with respect to the fine $C^{\infty}$ topology. Let $f$ be such a function. For any leafwise singularity $x$ of $f$, the jet $j^{k}\left(\left.f\right|_{F_{x}}\right)(x)$ has codimension $k-2$ at most. Thus, the germ of $\left.f\right|_{F_{x}}$ at $x$ is equivalent to $j^{k}\left(\left.f\right|_{F_{x}}\right)(x)$, and has finite codimension as well. Lemma 3.12 implies that the singularities of $\left.f\right|_{F_{x}}$ are isolated in $F_{x}$.

\section{3. $\mathcal{F}$-generic functions.}

Let $M$ be an $m$-dimensional manifold carrying a foliation $\mathcal{F}$ of dimension $n$ and codimension $q$.

Definition $3.22(\mathcal{F}$-genericity). A smooth real-valued function $f$ on $M$ is said to be $\mathcal{F}$-generic if $j^{1} f$ intersects $\Sigma_{\mathcal{F}}^{(n)}$ transversely and $j^{2} f$ intersects $\Sigma_{\mathcal{F}}^{\left(i_{1}, i_{2}\right)}$ transversely for all pairs $\left(i_{1}, i_{2}\right)$.

Definition 3.23 (Strong $\mathcal{F}$-genericity). A smooth real-valued function on $M$ is said to be strongly $\mathcal{F}$-generic if

i) the ordinary critical points of $f$ are both nondegenerate and leafwise nondegenerate;

ii) for every sequence $I$ of any length $k$, the map $j^{k} f$ intersects $\Sigma_{\mathcal{F}}^{I}$ transversely;

iii) the leafwise critical points of $f$ are isolated with respect to the leaf topology.

Here as well, it is a consequence of Thom's Transversality Theorem that the set of (strongly) $\mathcal{F}$-generic functions is residual in $C^{\infty}(M)$ for the fine $C^{\infty}$ topology. Indeed, the set of functions whose singularities are nondegenerate is a residual set (cf. [17]), as is the set of functions satisfying ii) 
(introduced in Definition 3.9), and the set of functions satisfying iii) (containing the set $\mathcal{B}$ introduced in Proposition 3.21). So the only thing that remains to be proven is that generically, critical points of $f$ are leafwise nondegenerate. Introduce the following subsets of $J^{2}(M, \mathbb{R})$

$$
W^{\ell} \stackrel{\text { def. }}{=} \Sigma^{(m, 0)} \cap \Sigma_{\mathcal{F}}^{(n, \ell)} \quad 1 \leq \ell \leq n .
$$

One easily proves that the $W^{\ell}$ 's are embedded submanifolds of $J^{2}(M, \mathbb{R})$ of codimension strictly larger than $m$. Hence, the set of functions whose second jet does not meet any $W^{\ell}$ is residual. By construction, the critical points of those functions are leafwise nondegenerate.

Remark 3.24. Reflecting on Definition 0.3, one might think that $c$ ) is superfluous. Indeed, the set of strongly $\mathcal{F}$-generic functions is dense in $C^{\infty}(M)$, and we can therefore approximate in the fine $C^{\infty}$ topology any given function by a strongly $\mathcal{F}$-generic one. The problem is that one usually creates leafwise local maxima in the process. It is not clear, although quite plausible, that a function $f$ with no leafwise local maxima always admits a nearby strongly $\mathcal{F}$-generic function with no leafwise local maxima. How-

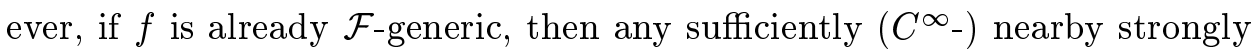
$\mathcal{F}$-generic function will not have leafwise local maxima either.

The proof of the next lemma is very similar to that of the corresponding result in the single-leaf case (cf. [17] for example). We nevertheless include it for the sake of completeness.

Lemma 3.25. Let $f: M \rightarrow \mathbb{R}$ be a smooth function, and let $x$ be a point in $M$ for which $j^{2} f(x) \in \Sigma_{\mathcal{F}}^{(n, n-r)}$. Then there exists a chart centered at $x$ and adapted to $\mathcal{F}$, with local coordinates $x_{1}, \ldots, x_{m}$, with respect to which $f$ has the following expression

$$
f\left(x_{1}, \ldots, x_{m}\right)= \pm x_{1}^{2} \pm \ldots \pm x_{r}^{2}+f\left(0, \ldots, 0, x_{r+1}, \ldots, x_{m}\right) .
$$

Proof. Consider local coordinates $x_{1}, \ldots, x_{m}$ centered at $x$ for which $\mathcal{F}$ is defined near $x$ by the equations $x_{n+1}=c_{n+1}, \ldots, x_{m}=c_{m}$, where $c_{1}, \ldots, c_{m}$ are constants . Since $j^{2} f(x) \in \Sigma_{\mathcal{F}}^{(n, n-r)}$, the function $\left.f\right|_{F_{x}}$ is singular at $x$, and we may assume (after eventually performing a linear change of the coordinates $\left.x_{1}, \ldots, x_{n}\right)$ that

$$
\left(\frac{\partial^{2} f}{\partial x_{i} \partial x_{j}}(0)\right)_{1 \leq i, j \leq r}=\left(\begin{array}{cc}
I_{d} & 0 \\
0 & -I_{r-d}
\end{array}\right) .
$$


One deduces from the implicit function theorem that there exists a neighborhood $U$ of 0 in $\mathbb{R}^{m-r}$, a neighborhood $U^{\prime}$ of 0 in $\mathbb{R}^{r}$, and a function $\alpha:(U, 0) \rightarrow\left(U^{\prime}, 0\right)$ such that, for every $i=1, \ldots, r$, and for $x$ in $U^{\prime} \times U$, one has

$$
\frac{\partial f}{\partial x_{i}}\left(x_{1}, \ldots, x_{m}\right)=0 \text { if and only if } \quad\left(x_{1}, \ldots, x_{r}\right)=\alpha\left(x_{r+1}, \ldots, x_{m}\right) .
$$

Use $\alpha$ to define new coordinates near $x$ as follows:

$$
\begin{cases}x_{i}^{\prime}=x_{i}-\alpha_{i}\left(x_{r+1}, \ldots, x_{m}\right) & \text { for } 1 \leq i \leq r \\ x_{j}^{\prime}=x_{j} & \text { for } j>r\end{cases}
$$

Now

$$
\frac{\partial f}{\partial x_{i}^{\prime}}\left(0, \ldots, 0, x_{r+1}^{\prime}, \ldots, x_{m}^{\prime}\right)=0 \quad \text { for } \quad 1 \leq i \leq r .
$$

The primes are omitted hereafter. A classical argument (cf. [17] Lemma 2.1.) shows that in a convex neighborhood of 0 , one has

$$
\begin{aligned}
& f\left(x_{1}, \ldots, x_{m}\right) \\
& =f\left(0, \ldots, 0, x_{r+1}, \ldots, x_{m}\right)+\sum_{1 \leq i \leq j \leq r} x_{i} x_{j} f_{i j}\left(x_{1}, \ldots, x_{m}\right) .
\end{aligned}
$$

Consider the coordinates $x_{1}^{\prime}, \ldots, x_{m}^{\prime}$ defined near $x$ by

$$
\left\{\begin{array}{l}
x_{1}^{\prime}=x_{1} \sqrt{\left|f_{11}(x)\right|}+\frac{1}{2} \sum_{i=2}^{r} x_{i} \frac{f_{1 i}(x)}{\sqrt{\left|f_{11}(x)\right|}} \\
x_{i}^{\prime}=x_{i} \text { for } i \geq 2 .
\end{array}\right.
$$

With respect to these coordinates, $f$ can be written as $(10)$, where $f_{11}(x)=$ 1 , where $f_{1 i}(x)=0$, and where $f_{i j}(0)$ remains unchanged. We repeat this process, modifying successively $x_{2}, \ldots, x_{r}$, until the expression (9) is achieved. Observe that every change of coordinates that has been made preserves $\mathcal{F}$ since it leaves the coordinates $x_{n+1}, \ldots, x_{m}$ untouched.

Proposition 3.26. Let $f: M \rightarrow \mathbb{R}$ be an $\mathcal{F}$-generic function without leafwise local maxima, and let $x$ be in $\Sigma_{\mathcal{F}, f}^{(n, n-r)}$. Then, for any system of coordinates centered at $x$ satisfying the conclusion of the previous lemma, the quadratic form $Q\left(x_{1}, \ldots, x_{r}\right)= \pm x_{1}^{2} \pm \ldots \pm x_{r}^{2}$ occurring in expression (9) must have at least one positive sign. 
Proof. Suppose on the contrary that either $Q=0$ or $Q$ is negative definite. We claim that, if so, any neighborhood of $x$ contains leafwise local maxima of $f$, contradicting the assumption.

Let $x \in M$, and let $(U, \varphi)$ be a chart at $x$, adapted to $\mathcal{F}$, with local coordinates $x_{1}, \ldots, x_{m}$, with respect to which $f$ is expressed by (9). The chart $(U, \varphi)$ induces a chart $(\tilde{U}, \tilde{\varphi})$ on $J^{2}(M, \mathbb{R})$ :

$$
\begin{aligned}
\tilde{\varphi}: \tilde{U}=\left(\pi^{2}\right)^{-1}(U) & \rightarrow \varphi(U) \times \mathbb{R} \times \mathbb{R}^{m} \times \mathbb{S}^{m} \\
p=j^{2} g(x) & \mapsto\left(\varphi(x), g(x), L_{p}, S_{p}\right),
\end{aligned}
$$

where $\pi^{2}$ denotes the natural projection $J^{2}(M, \mathbb{R}) \rightarrow M$, and where $\mathbb{S}^{m}$ denotes the set of symmetric $m \times m$ matrices with real coefficients. The symbol $L_{p}$ (respectively $S_{p}$ ) stands for the Jacobian (respectively Hessian) matrix of $g \circ \varphi^{-1}$ at $\varphi(x)$.

It is convenient to decompose a symmetric $n \times n$ matrix $S$ into blocks as follows:

$$
S=\left(\begin{array}{cc}
A & B \\
B^{t} & D
\end{array}\right)
$$

where $A$ and $D$ are $r \times r$ and $(n-r) \times(n-r)$ symmetric matrices, and where $B$ is some $r \times(n-r)$ matrix. If $A$ is nonsingular, then $S$ has rank $r$ if and only if the matrix $e(S)=-B^{t} A^{-1} B+D$ vanishes. Define $\mathcal{U}$ to be the neighborhood of $j^{2} f(x)$ in $\tilde{U}$ consisting of jets of local maps whose Hessian has a nonsingular upper left $r \times r$ block. Thus

$$
\Sigma_{\mathcal{F}}^{(n, n-r)} \cap \mathcal{U}=(\rho \circ \tilde{\varphi})^{-1}(0),
$$

where $\rho$ denotes the submersion:

$$
\begin{array}{lll}
\rho=\rho^{1} \times \rho^{2}: & \begin{array}{ll}
\tilde{\varphi}(\mathcal{U}) & \rightarrow \mathbb{R}^{n} \times \mathbb{S}^{n-r} \\
& (x, t, L, S)
\end{array} & \mapsto(p(L), e(q(S))),
\end{array}
$$

and where $p: \mathbb{R}^{n} \times \mathbb{R}^{m-n} \rightarrow \mathbb{R}^{n}$ (respectively $q: \mathbb{S}^{m} \rightarrow \mathbb{S}^{n}$ ) is the natural projection. Since $j^{2} f$ (respectively $j^{1} f$ ) intersects $\Sigma_{\mathcal{F}}^{(n, n-r)}$ (respectively $\Sigma_{f}$ ) transversely at $x$, the maps $\rho \circ \tilde{\varphi} \circ j^{2} f$ and $\rho^{1} \circ \tilde{\varphi} \circ j^{2} f$ are submersions near $x$. Moreover $\left(\rho^{1} \circ \tilde{\varphi} \circ j^{2} f\right) *_{x}\left(T_{x} \Sigma_{f}\right)=0$, and thus

$$
\left.\rho^{2} \circ \tilde{\varphi} \circ j^{2} f\right|_{\Sigma_{f} \cap U}: \Sigma_{f} \cap U \rightarrow \mathbb{S}^{n-r}
$$

is a submersion near $x$ as well. This implies in particular that for $\varepsilon$ sufficiently small, there exists a $x_{o}$ in $\Sigma_{f} \cap U$ such that $\rho^{2} \circ \tilde{\varphi} \circ j^{2} f\left(x_{o}\right)=-\varepsilon I_{n-r}$. Then $\bar{d}^{2} f\left(x_{o}\right)=-\mathrm{Id}$ is negative definite. Thus $f$ achieves a leafwise local maximum at $x_{o}$. 
Remark 3.27. The assumption that $f$ is $\mathcal{F}$-generic and has no leafwise local maxima prevents $f$ from having totally degenerate singularities (singularities at which the foliated second differential of $f$ vanishes).

\section{Leafwise gradient vector fields.}

Let $(M, \mathcal{F})$ be a foliated manifold, let $f$ be a positive, proper, strongly $\mathcal{F}$ generic function on $(M, \mathcal{F})$ (Definition 3.23), and let $g$ be a Riemannian metric on $M$.

Definition 4.1. The leafwise gradient vector field of $f$ associated to the metric $g$, denoted by $\nabla_{\mathcal{F}} f$, is the orthogonal projection onto $T \mathcal{F}$ of the (ordinary) gradient vector field of $f$. Equivalently, $\nabla_{\mathcal{F}} f$ is the section of $T \mathcal{F}$ whose restriction to each leaf $F$ is the gradient of $\left.f\right|_{F}$ associated to the metric $\left.g\right|_{F}$.

The local flow of $\nabla_{\mathcal{F}} f$ is denoted by $\varphi^{t}$. The orbit of a point $x$ under the flow $\varphi^{t}$ is denoted by $\theta_{x}$. If $S$ is some subset of $\mathbb{R}$, the portion $\left\{\varphi^{t}(x) ; t \in S\right\}$ of $\theta_{x}$ is denoted by $\varphi^{S}(x)$. Finally, the negative (respectively positive) limit set of the orbit $\theta_{x}$ is denoted by $\mathcal{L}_{x}^{-}$(respectively $\mathcal{L}_{x}^{+}$), i.e.,

$$
\mathcal{L}_{x}^{-}=\bigcap_{n=0}^{\infty} \overline{\varphi^{(-\infty,-n)}(x)} \quad \text { and } \quad \mathcal{L}_{x}^{+}=\bigcap_{n=0}^{\infty} \overline{\varphi^{(n, \infty)}(x)}
$$

\section{Lemma 4.2.}

i) The set of points where $\nabla_{\mathcal{F}} f$ vanishes coincides with $\Sigma_{f}$. Where $\nabla_{\mathcal{F}} f$ does not vanish, $\left(\nabla_{\mathcal{F}} f\right)(f)>0$.

ii) For every $x$ in $M$, the set $\mathcal{L}_{x}^{-}$reduces to a single point, lying in $\Sigma_{f}$. Similarly, the set $\mathcal{L}_{x}^{+}$is either empty if the trajectory of $x$ is unbounded, or consists also of a single point, lying in $\Sigma_{f}$ as well.

Proof.

i) Both assertions follow from the observation that for any $X$ in $T \mathcal{F}$, we have

$$
X f=d f(X)=g(\nabla f, X)=g\left(\nabla_{\mathcal{F}} f, X\right) .
$$

ii) Since $\varphi^{(-\infty, 0]}(x)$ is bounded $\left(\varphi^{(-\infty, 0]}(x) \subset f^{-1}[0, f(x)]\right)$, for any sequence $t_{k} \rightarrow-\infty$ of real numbers, the sequence $\varphi^{t_{k}}(x)$ has a converging 
subsequence . The set $\mathcal{L}_{x}^{-}$is therefore nonempty. Moreover, because $\mathcal{L}_{x}^{-}$is $\varphi^{t}$-invariant and contained in a level set of $f$ (the one at level $\inf \left\{f\left(\varphi^{t}(x)\right) ; t \in \mathbb{R}\right\}$ ), it must be contained in $\Sigma_{f}$ (since $f$ increases along nonconstant trajectories of $\left.\nabla_{\mathcal{F}} f\right)$.

Observe now that $\mathcal{L}_{x}^{-}$is connected. Indeed, suppose it is not, and let $O_{1}$ and $O_{2}$ be disjoint open subsets of $M$ containing $\mathcal{L}_{x}^{-}$in their union. There exists a $T \leq 0$ for which $\varphi^{t}(x) \in O_{1} \cup O_{2}$ as soon as $t \leq T$. Otherwise there would be a sequence $t_{k} \rightarrow-\infty$ for which $O_{1} \cup O_{2}$ does not contain $\varphi^{t_{k}}(x)$, hence not either $\lim _{k \rightarrow \infty} \varphi^{t_{k}}(x)$. Connectedness of $\varphi^{(-\infty, T]}(x)$ implies that $\mathcal{L}_{x}^{-}$is entirely contained in one of the $O_{i}$ 's.

To conclude, we need to prove that $\mathcal{L}_{x}^{-}$is contained in the leaf $F_{x}$. Suppose not, and let $w$ be an point in $\mathcal{L}_{x}^{-}$that does not belong to $F_{x}$. Let also $(U, \varphi)$ be a chart adapted to $\mathcal{F}$ centered at $w$ with $\varphi(U)=$ $[0,1]^{n} \times[0,1]^{q}$. Denote by $P_{a}$ the plaque $\varphi^{-1}\left([0,1]^{n} \times\{a\}\right)$. Since $f$ is strongly $\mathcal{F}$-generic, its leafwise critical points are isolated for the leaf topology. We may therefore assume that $w$ is the only point in $\Sigma_{f}$ that lies in $P_{0}$. Because $w$ belongs to $\mathcal{L}_{x}^{-}-F_{x}$, there is a sequence $a_{k}$ converging to 0 such that each plaque $P_{a_{k}}$ contains a segment $\varphi^{\left[t_{k}, s_{k}\right]}(x)$ of $\theta_{x}$ that meets the boundary of $P_{a_{k}}$ along its endpoints (i.e. $\varphi^{t_{k}}(x)$ and $\varphi^{s_{k}}(x)$ are in $\partial P_{a_{k}}$ ). We may also assume, without loss of generality, that $t_{k}$ and $s_{k}$ both converge to $-\infty$. Now the sequence $\varphi^{t_{k}}(x)$ has a subsequence that converges to a point $w^{\prime}$ in $\partial P_{0}$. Since by construction $w^{\prime}$ lies in $\mathcal{L}_{x}^{-}$, it is necessarily a leafwise critical point of $f$, contradicting the hypothesis made earlier on $U$.

We have now all the ingredients needed : $\mathcal{L}_{x}^{-}$is nonempty and connected, consists of leafwise critical points of $f$, and is entirely contained in a leaf. Property iii) of Definition 3.23 implies thus that $\mathcal{L}_{x}^{-}$is a single point. The same argument proves the second part of statement $i i$ ) as well.

Definition 4.3. Given $x$ in $M$, the limit point $\mathcal{L}_{x}^{-}$is denoted by $x^{-}$. Similarly, when $\theta_{x}$ in bounded, the limit point $\mathcal{L}_{x}^{+}$is denoted by $x^{+}$.

\section{Definition 4.4.}

1. If $A$ is a subset of $\Sigma_{f}$, we define the stable set of $A$ to be the set $\mathcal{W}(A)=\left\{x \in M ; x^{+} \in A\right\}$. 
2. If $A$ is a subset of $M$, the saturation of $A$ is defined to be the set $\mathcal{S} A=\left\{x \in M ; \varphi^{t}(x) \in A\right.$ for some $\left.t \geq 0\right\}$.

The following lemma will reduce the proof that the h-principle holds on $M$ to the proof that it holds near the "skeleton" $\mathcal{W}\left(\Sigma_{f}\right)$. Let $(a, b)$ be any pair of real numbers with $a<b$. The slice $f^{-1}([a, b])$ is denoted hereafter by $M_{a}^{b}$, and the set $\mathcal{W}\left(\Sigma_{f} \cap M_{a}^{b}\right) \cap M_{a}^{b}$ by $\mathcal{W}_{a}^{b}\left(\Sigma_{f}\right)$.

Lemma 4.5. Given any pair $a<b$ of regular values of $f$, and given any neighborhood $U$ of $M_{0}^{a} \cup \mathcal{W}_{a}^{b}\left(\Sigma_{f}\right)$, the flow of $\nabla_{\mathcal{F}} f$ yields a foliated isotopy $\psi_{t}: M \rightarrow M, t \in[0,1]$, such that

- $\psi_{t}\left(M_{0}^{b}\right) \subset M_{0}^{b}$ for all $t$ in $[0,1]$,

- $\psi_{1}\left(M_{0}^{b}\right) \subset U$,

- $\psi_{t}$ coincides with the identity map on $M_{0}^{a} \cup \mathcal{W}_{a}^{b}\left(\Sigma_{f}\right)$,

- $\psi_{t}$ is stationary for $t \geq \frac{1}{2}$.

Proof. Let $U$ be an open neighborhood of $M_{0}^{a} \cup \mathcal{W}_{a}^{b}\left(\Sigma_{f}\right)$. As a first step, we prove existence of a neighborhood $N$ of $\Sigma_{f} \cap M_{a}^{b}$ whose saturation $\mathcal{S} N$ is contained in $U$. Suppose that such an $N$ does not exist. Consider the neighborhoods $N_{k}$ of $\Sigma_{f} \cap M_{a}^{b}$ given by $N_{k}=\{x \in M$; the distance between $x$ and $\Sigma_{f} \cap M_{a}^{b}$ is less than $\left.\frac{1}{k}\right\}$. Since $\Sigma_{f} \cap M_{a}^{b}$ is compact, $N_{k}$ is in $U$ for $k$ large enough. Now, suppose that for every $k$ there exists $x_{k}$ in $N_{k}$ such that for some negative $t_{k}$, the point $y_{k}=\varphi^{t_{k}}\left(x_{k}\right)$ does not belong to $U$. There exists subsequences $x_{k_{l}}$ and $y_{k_{l}}$ of $x_{k}$ and $y_{k}$ respectively, converging to points $x$ and $y$ respectively. The point $x$ lies in $\Sigma_{f} \cap M_{a}^{b}$, and the point $y$ lies outside $U$. Thus the trajectory $\theta_{y}$ intersects $f^{-1}(b)-\Sigma_{f}$ nontrivially. Denote by $\mathcal{H}$ the foliation of $M-\Sigma_{f}$ by the flow lines of $\nabla_{\mathcal{F}} f$. Let $\mathcal{K}$ be the portion of $\theta_{y}$ located between levels $f(y)$ and level $b$. Since $\mathcal{K}$ is a compact subset of a leaf of $\mathcal{H}$, there exists a closed neighborhood $O$ of $\mathcal{K}$ in $M-\Sigma_{f}$ such that $\left.\mathcal{H}\right|_{O}$ is isomorphic to a product foliation. For $l$ sufficiently large, $y_{k_{l}}$ is contained in $O$, and therefore $x_{k_{l}}$ and $x$ are contained in $O$ as well. This contradicts the hypothesis that $O$ does not intersect $\Sigma_{f}$.

Now choose an open neighborhood $N$ of $\Sigma_{f} \cap M_{a}^{b}$ for which $\mathcal{S} N \subset U$. Notice that $\mathcal{S} N$ is an open neighborhood of $\mathcal{W}_{a}^{b}\left(\Sigma_{f}\right)$. Since $f^{-1}(a)$ is compact, there exists a $\delta>0$ such that $f^{-1}([0, a+\delta)) \subset U$. Let $\tilde{U}=\mathcal{S} N \cup f^{-1}([0, a+\delta))$. Because $f$ increases along nonconstant trajectories of $\nabla_{\mathcal{F}} f$, there exists, for every $x$ in $M_{a}^{b}$, a $t_{o} \leq 0$ such that $\varphi^{t}(x) \in \tilde{U}$ 
for all $t \leq t_{o}$. Moreover, since $M_{a}^{b}$ is compact, such a $t_{o}$ can be chosen that does not depend on $x$. Hence $\varphi^{t_{o}}$ shrinks $M_{0}^{b}$ inside $\tilde{U}$, but does not coincide with the identity on $M_{0}^{a} \cup \mathcal{W}_{a}^{b}\left(\Sigma_{f}\right)$. Multiplying $\nabla_{\mathcal{F}} f$ by a smooth function $\eta: M \rightarrow[0,1]$ that equals 0 on $M_{0}^{a} \cup \mathcal{W}_{a}^{b}\left(\Sigma_{f}\right)$ and 1 outside $\tilde{U}$ yields a vector field $\eta \nabla_{\mathcal{F}} f$ whose flow provides us with the desired isotopy. Indeed, let $\psi_{t}=\varphi^{\theta(t) t_{o}}$, where $\theta$ is a smooth function $[0,1] \rightarrow[0,1] \mathrm{such}$ that $\theta(0)=0$ and $\theta(t)=1$ for all $t \geq \frac{1}{2}$. The isotopy $\psi_{t}$ satisfies the four required properties.

\section{Construction of the metric.}

Let $M$ be an $m$-dimensional manifold endowed with a foliation $\mathcal{F}$ of dimension $n$ and codimension $q$. Supposing the foliated manifold $(M, \mathcal{F})$ uniformely open (Definition 0.3 ), let $f: M \rightarrow \mathbb{R}$ be a strongly $\mathcal{F}$-generic function (Definition 3.23) without leafwise local maxima (cf. Remark 3.24). In this section, we carry out the construction of a Riemannian metric for which the stable set of the foliated singular locus of $f$ (Definition 3.2 and Definition 4.4) is locally contained in a finite union of embedded submanifolds, of codimension at least one, transverse to the foliation $\mathcal{F}$.

A submanifold $S$ of $M$ is said to intersect $\mathcal{F}$ transversely if for all $s$ in $S$, the quantity $\operatorname{dim}\left(T_{s} S+T_{s} \mathcal{F}\right)$ is maximal, that is, coincides with $\min \{\operatorname{dim} S+\operatorname{dim} \mathcal{F}, \operatorname{dim} M\}$. Observe that a submanifold $S$ whose dimension is strictly less than $q$ does never intersect each leaf of $\mathcal{F}$ transversely! Nevertheless, it might intersect the foliation $\mathcal{F}$ transversely. Let $g$ be a Riemannian metric on $M$. Recall the foliated second differential $\bar{d}^{2} f$ of $f$ (Subsection 3.1, formula (8)). Because the bilinear map $\bar{d}^{2} f(\sigma)$ is symmetric for all $\sigma \in \Sigma_{f}$, the identity $\bar{d}^{2} f(\sigma)(X, Y)=g(X, A Y)$ defines a $g$-self-adjoint map $A: T_{\sigma} \mathcal{F} \rightarrow T_{\sigma} \mathcal{F}$. The map $A$ can be diagonalized by means of a $g$ orthogonal basis. We therefore have a splitting $T_{\sigma} \mathcal{F}=V_{\sigma}^{+} \oplus V_{\sigma}^{-} \oplus V_{\sigma}^{0}$ into positive, negative and null eigenspaces for $A$. The space $V_{\sigma}^{0}$ is the kernel of $\bar{d}^{2} f(\sigma)$ and is thus intrinsically defined (independently of $g$ ), unlike the other two. The distributions $V^{ \pm}: \sigma \in \Sigma_{f} \mapsto V_{\sigma}^{ \pm}$, although not of constant rank, are smooth (unlike the distribution $V^{0}: \sigma \in \Sigma_{f} \mapsto V_{\sigma}^{0}$ ). Smoothness for such a distribution is defined as follows : for every $\sigma$ in $\Sigma_{f}$ there exist local sections $X_{1}, \ldots, X_{d}$ of $T M$ defined in a neighborhood $U$ of $\sigma$ in $\Sigma_{f}$ such that

- $\left\{X_{1}(\sigma), \ldots, X_{d}(\sigma)\right\}$ is a basis of $V_{\sigma}^{ \pm}$,

- $X_{i}\left(\sigma^{\prime}\right)$ belongs to $V_{\sigma^{\prime}}^{ \pm}$for all $\sigma^{\prime}$ in $U$. 
Remark 5.1. Consider a sequence $I=\left(i_{1}, \ldots, i_{k-1}, i_{k}=0\right)$. The rank of $V^{0}$ and hence of $V^{+} \oplus V^{-}$is constant on the submanifold $\Sigma_{\mathcal{F}, f}^{I}$ (cf. Section 3.1). Moreover, since both $V^{+}$and $V^{-}$are smooth distributions, their rank must be constant on any connected component of $\Sigma_{\mathcal{F}, f}^{I}$.

Definition 5.2. The union of the connected components of $\Sigma_{\mathcal{F}, f}^{I}$ on which $\operatorname{rk} V^{+}$achieves a fixed value $d$ is denoted by $\Sigma_{d}^{I}$, and called hereafter a stratum of $\Sigma_{f}$ (provided it is nonempty). We introduce an order on the set of strata as follows:

$$
\Sigma_{e}^{\left(j_{1}, \ldots, j_{\ell}\right)} \leq \Sigma_{d}^{\left(i_{1}, \ldots, i_{k}\right)} \Longleftrightarrow\left(n-e, j_{1}, \ldots, j_{\ell}\right) \geq\left(n-d, i_{1}, \ldots, i_{k}\right),
$$

where $\geq$ refers to the lexicographical order on tuples of nonnegative integers.

Observe that because $i_{k}=0$, a stratum $S$ is an embedded submanifold (generally neither connected nor closed) that intersects $\mathcal{F}$ transversely, in the sense that $T_{s} S \cap T_{s} \mathcal{F}=\{0\}$ for all $s$ in $S$. Moreover, there are only finitely many strata, and they form a partition $\Sigma_{f}$. Furthermore, the closure of $\Sigma_{d}^{I}$ is contained in the union of the $\Sigma_{e}^{J}$ 's with $e \leq d$ and $J \geq I$ (see end of Section 3.1), i.e., it is contained in the union of the smaller (with respect to the order introduced in Definition 5.2) strata. Consequently, the union of the $l$ smallest strata is a closed set.

\subsection{Outline of the construction of the metric.}

The construction is based on the following observation. Let $\sigma$ be an element of $\Sigma_{f}$, and let $x_{1}, \ldots, x_{m}$ be local coordinates defined on a neighborhood $U$ of $\sigma$ in $M$ with respect to which $f$ has the following expression:

$$
f\left(x_{1}, \ldots x_{m}\right)=x_{1}^{2}+\ldots+x_{d}^{2}+f\left(0, \ldots, 0, x_{d+1}, \ldots, x_{m}\right),
$$

with $d>0$ (Lemma 3.25 and Proposition 3.26). Let us introduce the following piece of notation : given an open subset $O$ of $M$, let

$$
\mathcal{W}_{O}\left(\Sigma_{f}\right)=\left\{x \in O ; x^{+} \in \Sigma_{f} \cap O \text { and } \varphi^{t}(x) \in O \quad \forall t \geq 0\right\}
$$

where $\varphi^{t}$ denotes the flow of the leafwise gradient vector field $\nabla_{\mathcal{F}} f$ of $f$ associated to the metric $g$ (Definition 4.1), and where $x^{+}=\lim _{t \rightarrow \infty} \varphi^{t}(x)$ (cf. Definition 4.3). The set $\mathcal{W}_{O}\left(\Sigma_{f}\right)$ is the stable set (Definition 4.4) of $\Sigma_{f} \cap O$ with respect to $\left.\left(\nabla_{\mathcal{F}} f\right)\right|_{O}$. If instead of the metric $g$, we use the 
Euclidean metric with respect to the coordinates $x_{1}, \ldots, x_{m}$ to construct $\nabla_{\mathcal{F}} f$, then

$$
\mathcal{W}_{U}\left(\Sigma_{f}\right) \subset\left\{x_{1}=0, \ldots, x_{d}=0\right\}
$$

and the plane $\left\{x_{1}=0, \ldots, x_{d}=0\right\}$ is transverse to $\mathcal{F}$, as needed.

The problem is that it is not clear that a global Riemannian metric $g$ exists with the property that every point $\sigma$ in $\Sigma_{f}$ admits local coordinates as above, with respect to which $g$ is Euclidean (at least along the leaves). Fortunately, we do not need that much: for the property (11) to hold, it is sufficient to have a Riemannian metric on $U$ for which the plane $\left\{x_{1}=\right.$ $\left.0, \ldots, x_{d}=0\right\}$ is orthogonal to the planes $\left\{x_{d+1}=c_{d+1}, \ldots, x_{m}=c_{m}\right\}$ (cf. Proposition 5.3). This constitutes the key observation, as it can be globalized, at least to a neighborhood of a stratum. Subsection 5.2 shows how. Briefly, starting with the (constant rank) distribution $V^{+}$on a stratum $S$, we construct a foliation $\mathcal{G} \subset \mathcal{F}$ on a neighborhood $\mathcal{N}$ of $S$ such that $T_{S} \mathcal{G}=$ $V^{+}$(the foliation $\mathcal{G}$ plays the role of the planes $\left\{x_{d+1}=c_{d+1}, \ldots, x_{m}=c_{m}\right\}$ ). Then, we consider the manifold $P$ of critical points of $f$ along the leaves of $\mathcal{G}$ (the manifold $P$ plays the role of the plane $\left\{x_{1}=0, \ldots, x_{d}=0\right\}$ ). It is shown that any metric for which $T P$ is orthogonal to $T_{P} \mathcal{G}$ has the property that $\mathcal{W}_{\mathcal{N}}\left(\Sigma_{f}\right) \subset P$ (after eventually reducing $\mathcal{N}$ slightly).

Going from one stratum to the entire $\Sigma_{f}$ is done by induction on the order of the strata (Subsection 5.3). Basically, to carry out this induction, we need to construct the foliation $\mathcal{G}$ in such a way that it "matches" the foliations already constructed near smaller order strata, meaning that where two such foliations coexist, the largest dimensional one contains the other one. The ordering is, roughly speaking, according to the number of strata that a given stratum contains in its closure. Closed strata are the smallest, strata having only closed strata in their closure come next, and so forth. This is dictated by the lexicographical ordering of the Boardman symbols, combined with the rank of the distribution $V^{+}$. Once a "coherent" set of foliations has been constructed, one may build a metric for which $\mathcal{W}_{\mathcal{N}}\left(\Sigma_{f}\right) \subset P$ for all strata $S$ (cf. Subsection 5.4).

\subsection{The metric near one stratum.}

Let $S$ be a (neither necessarily connected nor closed) embedded submanifold of $\Sigma_{f}$. Suppose that $S$ intersects $\mathcal{F}$ transversely, and that the dimension of $V^{+}$is constant on $S$. Typically $S$ is a stratum of $\Sigma_{f}$ (cf. Definition 5.2). Consider also exp : $\tilde{\mathcal{O}} \subset T M \rightarrow M$, the exponential map associated to $g$, 
defined on a fiberwise convex neighborhood $\tilde{\mathcal{O}}$ of the 0 -section in $T M$. The construction of the metric near $S$ is divided into the five following steps.

First step. The submanifold $S$ is extended to an embedded submanifold $S^{\prime}$ of $M$, transverse to $\mathcal{F}$, and of dimension $q=\operatorname{cod} \mathcal{F}$.

If $E=\left(T S+T_{S} \mathcal{F}\right)^{\perp}$, then $\left.\exp \right|_{\tilde{\mathcal{O}} \cap E}$ is an embedding provided $\mathcal{O}=$ $\tilde{\mathcal{O}} \cap E$ is sufficiently small. Define $S^{\prime}=\exp (\mathcal{O})$. Because $\exp _{*}=$ Id along the 0-section, the bundle $T_{S} S^{\prime}$ coincides with $T S \oplus E$. In particular, the submanifold $S^{\prime}$ is transverse to $\mathcal{F}$ along $S$. We may assume that $\mathcal{O}$ has been chosen small enough for $S^{\prime}$ to be transverse to $\mathcal{F}$ everywhere.

Second step. Let $\mathcal{D}$ be a subbundle of $T_{S} \mathcal{F}$ (e.g., $\mathcal{D}=V^{+}$when $S$ is a stratum). We extend $\mathcal{D}$ to a subbundle $\mathcal{D}^{\prime}$ of $T_{S^{\prime}} \mathcal{F}$.

Let $\nabla$ be a linear connection on $T_{S^{\prime}} \mathcal{F}$. For every $x$ in $S^{\prime}$, there is a natural path between $x$ and a point $x_{o}$ in $S$. Indeed, take $\gamma_{x}:[0,1] \rightarrow S^{\prime}$ : $t \mapsto \exp (t X)$, where $\exp (X)=x$. The connection $\nabla$ and the path $\gamma_{x}$ induce a linear isomorphism $i_{x}: T_{x_{o}} \mathcal{F} \rightarrow T_{x} \mathcal{F}$, obtained by parallel translation along $\gamma_{x}$ with respect to $\nabla$. For $x$ in $S^{\prime}$, define $\mathcal{D}_{x}^{\prime}$ to be $i_{x}\left(\mathcal{D}_{x_{o}}\right)$.

Third step. Given an embedded submanifold $S^{\prime}$, complementary to $\mathcal{F}$, and a subbundle $\mathcal{D}^{\prime}$ of $T_{S^{\prime}} \mathcal{F}$, we extend $\mathcal{D}^{\prime}$ to a smooth foliation $\mathcal{G}$ tangent to $\mathcal{F}$, defined on a tubular neighborhood $\mathcal{N}$ of $S^{\prime}$.

Consider the map

$$
\xi: \mathcal{U} \subset T_{S^{\prime}} \mathcal{F} \rightarrow M: X_{s} \in T_{s} \mathcal{F} \mapsto \tau_{X_{s}}(1)
$$

where $\tau_{X_{s}}$ denotes the geodesic for the Riemannian metric $\left.g\right|_{F_{s}}$ starting at $s$ and tangent to $X_{s}$ (in particular $\tau_{X_{s}} \subset F_{s}$ ), and where $\mathcal{U}$ is some fiberwise convex neighborhood of the 0 -section on which $\xi$ is an embedding. The map $\xi$ is called hereafter the foliated exponential map associated to the metric $g$. Consider now the foliation $\tilde{\mathcal{G}}$ of $\mathcal{U}$ by the traces of the cosets of $\mathcal{D}^{\prime}$, and the push-forward $\mathcal{G}$ of $\tilde{\mathcal{G}}$ via the map $\xi$. Let $\mathcal{N}=\xi(\mathcal{U})$.

Fourth step. Suppose that the foliated second differential $\bar{d}^{2} f$ of $f$ is positive definite on $\mathcal{D}$. Let $P$ be the foliated singular locus of $f$ with respect to the foliation $\mathcal{G}$, that is, the set $\left\{x \in \mathcal{N} ; d f\left(T_{x} \mathcal{G}\right)=0\right\}$. Provided the neighborhood $\mathcal{N}$ of $S$ is sufficiently small, the set $P$ is an embedded submanifold of $\mathcal{N}$ transverse to $\mathcal{G}$ and closed in $\mathcal{N}$.

Let $p: B T \mathcal{G} \rightarrow \mathcal{N}$ be the bundle of basis of $T \mathcal{G}$. Define the map

$$
\eta: B T \mathcal{G} \rightarrow \mathbb{R}^{d}:\left\{e_{1}, \ldots, e_{d}\right\} \mapsto\left(d f\left(e_{1}\right), \ldots, d f\left(e_{d}\right)\right),
$$


where $d$ is the dimension of $\mathcal{G}$. Since $\bar{d}^{2} f$ is nondegenerate on $\mathcal{D}=T_{S} \mathcal{G}$, the map $\eta$ is a submersion near $p^{-1}(S)$. Therefore, the map $\eta$ is a submersion near $\eta^{-1}(0)$ provided $\mathcal{N}$ is sufficiently small. The submanifold $\eta^{-1}(0)$ coincides with $p^{-1}(P)$, where $P$ is a closed embedded submanifold of $\mathcal{N}$. To prove that $P$ is transverse to $\mathcal{G}$, consider a vector $X$ in $T_{p} P \cap T_{p} \mathcal{G}$, with $p$ in $P$. Extend $X$ to a section $\tilde{X}$ of $T \mathcal{G}$ defined on a neighborhood $U$ of $p$ in $\mathcal{N}$. On $P \cap U$, the function $\tilde{X} f$ vanishes, and because $X$ is tangent to $P$, we have $0=X(\tilde{X} f)=\bar{d}^{2} f(X, X)$ (notice that $\bar{d}^{2} f$ is indeed well-defined on $T_{P} \mathcal{G}$; it is the foliated second differential of $f$ with respect to the foliation $\mathcal{G})$. Since $\bar{d}^{2} f$ is nondegenerate on $T_{S} \mathcal{G}=\mathcal{D}$, it remains nondegenerate on $T_{P} \mathcal{G}$ provided $\mathcal{N}$ is sufficiently small. Thus, the vector field $X$ must vanish.

Fifth step. Let $h$ be a Riemannian metric for which $T P$ is orthogonal to $T_{P} \mathcal{G}$. Such a metric can be constructed by means of a partition of unity for instance. The leafwise gradient vector field $\nabla_{\mathcal{F}} f$ of $f$ associated to $h$ (Definition 4.1) satisfies the the following property.

Proposition 5.3. There exists a neighborhood $\mathcal{M}$ of $\mathcal{N} \cap \Sigma_{f}$ contained in $\mathcal{N}$ for which

$$
\mathcal{W}_{\mathcal{M}}\left(\Sigma_{f}\right) \subset P
$$

Proof. First observe that if $x$ belongs to $P$, then $\left(\nabla_{\mathcal{F}} f\right)_{x}$ belongs to $T P$ (as implied by the identity $\left.h\left(\nabla_{\mathcal{F}} f, T_{x} \mathcal{G}\right)=d f(x)\left(T_{x} \mathcal{G}\right)=0\right)$. We will then show that for some sufficiently small neighborhood $\mathcal{M}$ of $\mathcal{N} \cap \Sigma_{f}$, no trajectory of $\left.\left(\nabla_{\mathcal{F}} f\right)\right|_{\mathcal{M}}$ starting outside $P \cap \mathcal{M}$ can approach $P \cap \mathcal{M} \supset \Sigma_{f}$ in forward time.

Since $\mathcal{G} \subset \mathcal{F}$, and since $P$ is complementary to $\mathcal{G}$, given $x$ in $P$, there exist coordinates $x_{1}, \ldots, x_{m}$, called nice hereafter, on a neighborhood $U$ of $x$ such that

i) $x_{n+1}=c_{n+1}, \ldots, x_{m}=c_{m}$ define $\left.\mathcal{F}\right|_{U}$,

ii) $x_{d+1}=c_{d+1}, \ldots, x_{m}=c_{m}$ define $\left.\mathcal{G}\right|_{U}$,

iii) $x_{1}=0, \ldots, x_{d}=0$ define $P \cap U$,

iv) $f\left(x_{1}, \ldots, x_{m}\right)=x_{1}^{2}+\ldots+x_{d}^{2}+f\left(0, \ldots, 0, x_{d+1}, \ldots, x_{m}\right)$.

The proof of this fact, very similar to that of Lemma 3.25, is omitted.

Let $x$ be an element of $P$ and let $x_{1}, \ldots, x_{m}$ be nice coordinates defined on a neighborhood $U$ of $x$. Let also $u=\sum_{i=1}^{d} u_{i} \frac{\partial}{\partial x_{i}}$ be a nonvanishing constant 
vector field on $U$ tangent to the foliation $\mathcal{G}$. In the next paragraph, a dot - will denote the Euclidean scalar product with respect to the coordinates $x_{1}, \ldots, x_{m}$. We have the following sequence of identities:

$$
\begin{aligned}
\frac{\partial}{\partial u}\left(\nabla_{\mathcal{F}} f \cdot u\right)(x) & =\sum_{i, j=1}^{d} u_{i} \frac{\partial}{\partial x_{i}}\left(\sum_{k=1}^{n} \frac{\partial f}{\partial x_{k}} h^{k j} u_{j}\right)(x) \\
& =\sum_{i, j=1}^{d} \sum_{k=1}^{n} u_{i} u_{j}\left(\frac{\partial^{2} f}{\partial x_{i} \partial x_{k}}(x) h^{k j}(x)+\frac{\partial f}{\partial x_{k}}(x) \frac{\partial h^{k j}}{\partial x_{i}}(x)\right) \\
& =2 h(u, u)(x)+\sum_{i, j=1}^{d} \sum_{k=1}^{n} u_{i} u_{j} \frac{\partial f}{\partial x_{k}}(x) \frac{\partial h^{k j}}{\partial x_{i}}(x),
\end{aligned}
$$

where $h^{k j}$ denotes the component $k, j$ of the inverse of the matrix of $\left.h\right|_{T \mathcal{F} \times T \mathcal{F}}$. In particular, if $x$ belongs to $P \cap \Sigma_{f}=\mathcal{N} \cap \Sigma_{f}$, we have

$$
\frac{\partial}{\partial u}\left(\nabla_{\mathcal{F}} f \cdot u\right)(x)=2 h(u, u)(x)>0 .
$$

Hence, for all $x$ in $P \cap \Sigma_{f}$, there exists an open neighborhood $U_{x}$ of $x$, endowed with nice coordinates, such that $\frac{\partial}{\partial u}\left(\nabla_{\mathcal{F}} f \cdot u\right)$ is strictly positive on $U_{x}$, for all nonvanishing constant vector field $u$ tangent to the foliation $\mathcal{G}$. As a consequence, a trajectory $\theta_{x}$ of $\nabla_{\mathcal{F}} f$ for which $\theta_{x}(t)$ lies in $U_{x}-\left(P \cap U_{x}\right)$ for all $t$ in $\left[t_{o}, \infty\right)$ cannot converge to a point in $P \cap U_{x}$. Indeed, Let $w$ be any point in $U_{x}-\left(P \cap U_{x}\right)$, and let $u$ be a nonvanishing constant vector field parallel to the vector joining $w_{o}=\left(0, \ldots, 0, x_{d+1}, \ldots, x_{m}\right)$ to $w=$ $\left(x_{1}, \ldots, x_{m}\right)$. Then, since $\left.\frac{\partial}{\partial u}\left(\nabla_{\mathcal{F}} f \cdot u\right)\right|_{U_{x}}>0$ and $\left(\nabla_{\mathcal{F}} f \cdot u\right)\left(w_{o}\right)=0$, the quantity $\left(\nabla_{\mathcal{F}} f \cdot u\right)(w)$ is strictly positive. Supposing $w=\theta_{x}(t)$ for some $t \geq t_{o}$, the last assertion implies that the Euclidean distance between $\theta_{x}(t)$ and $P \cap U_{x}$ grows with $t$.

Let $\left\{U_{i} ; i \geq 1\right\}$ be a locally finite refinement of the covering $\left\{U_{x} ; x \in\right.$ $\left.P \cap \Sigma_{f}\right\}$ of $P \cap \Sigma_{f}$. Let $\mathcal{M}=\mathcal{N} \cap\left(\cup_{i \geq 1} U_{i}\right)$. Any trajectory of $\left.\left(\nabla_{\mathcal{F}} f\right)\right|_{\mathcal{M}}$ starting in $P \cap \mathcal{M}$ remains in $P \cap \mathcal{M}$, and no trajectory starting outside $P \cap \mathcal{M}$ will approach $P \cap \mathcal{M}$ in forward time. Indeed, suppose on the contrary that $\lim _{t \rightarrow \infty} \theta_{x}(t) \in P$, for some trajectory $\theta_{x}$ of $\left.\left(\nabla_{\mathcal{F}} f\right)\right|_{\mathcal{M}}$. Then, for some $t_{o}$ and some $i$, the point $\theta_{x}(t)$ lies in $U_{i}$ for all $t \geq t_{0}$. The discussion in the previous paragraph implies that $\theta_{x}(t) \in P$ for all $t \geq t_{0}$, and hence that $\theta_{x} \subset P$.

Remark 5.4. The reason for introducing $\mathcal{M}$ instead of supposing once more $\mathcal{N}$ small enough is that we need to make sure that the portion of 
$\Sigma_{f}$ whose stable set is taken care of $\left(\mathcal{N} \cap \Sigma_{f}\right.$ in the previous proposition) is fixed once $\mathcal{G}$ is given.

Conclusion 5.5. Suppose given the following data:

- an embedded submanifold $S$ of $\Sigma_{f}$ intersecting $\mathcal{F}$ transversely,

- a subbundle $\mathcal{D}$ of $T_{S} \mathcal{F}$ restricted to which $\bar{d}^{2} f$ is positive definite.

We can then construct a foliation $\mathcal{G}$ tangent to $\mathcal{F}$, defined on a tubular neighborhood $\mathcal{N}$ of $S$, satisfying the following properties.

i) $T_{S} \mathcal{G}$ coincides with $\mathcal{D}$.

ii) The foliated singular locus $P$ of $f$ with respect to the foliation $\mathcal{G}$, is an embedded submanifold transverse to $\mathcal{G}$ (equivalently $\bar{d}^{2} f$ is positive definite on $\left.T_{P} \mathcal{G}\right)$. In particular, $P$ as codimension at least one and intersects $\mathcal{F}$ transversely.

iii) Let $h$ be a Riemannian metric on $\mathcal{N}$ for which $T P$ is perpendicular to $T_{P} \mathcal{G}$. Then there exists a neighborhood $\mathcal{M}$ of $\mathcal{N} \cap \Sigma_{f}$ contained in $\mathcal{N}$ such that the leafwise gradient vector field of $f$ associated to $h$ satisfies the property that $\mathcal{W}_{\mathcal{M}}\left(\Sigma_{f}\right) \subset P$.

\subsection{Construction of a complete system of foliations.}

As explained in the beginning of Section 5, the construction of a suitable Riemannian metric goes by defining a collection of foliations, one near each stratum, in a compatible way. The word compatible means that where two such foliations coexist, the one whose dimension is largest contains the other one. This construction is the subject of the present subsection.

Definition 5.6. Consider a collection of strata $S_{1}, \ldots, S_{l}$ satisfying the property that for all $k$, the set $S_{k} \cup \ldots \cup S_{l}$ is closed in $M$. A system of foliations on $\cup_{k=1}^{l} S_{k}$ is a collection $\left\{\mathcal{G}_{k} ; k=1, \ldots, l\right\}$ of foliations, where $\mathcal{G}_{k}$ is defined on a tubular neighborhood $\mathcal{N}_{k}$ of an open subset $O_{k}=\mathcal{N}_{k} \cap S_{k}$ of $S_{k}$. Moreover, the following properties are required to hold :

a) For all $1 \leq k \leq l, S_{k} \subset \mathcal{N}_{k} \cup \ldots \cup \mathcal{N}_{l}$.

b) If $S_{k_{1}}$ and $S_{k_{2}}$ are such that $\bar{S}_{k_{1}} \cap S_{k_{2}}=\emptyset=S_{k_{1}} \cap \bar{S}_{k_{2}}$ then $\mathcal{N}_{k_{1}} \cap \mathcal{N}_{k_{2}}=$ $\emptyset$.

c) $\operatorname{dim} \mathcal{G}_{k}=\operatorname{rk}\left(\left.V^{+}\right|_{S_{k}}\right)$. 
d) If $\operatorname{dim} \mathcal{G}_{k_{1}} \geq \operatorname{dim} \mathcal{G}_{k_{2}}$, then $T \mathcal{G}_{k_{1}} \supset T \mathcal{G}_{k_{2}}$ on $\mathcal{N}_{k_{1}} \cap \mathcal{N}_{k_{2}}$.

e) For all $k$, the foliated singular locus of $f$ with respect to $\mathcal{G}_{k}$, i.e. the set $P_{k}=\left\{p \in \mathcal{N}_{k} ; d f(p)\left(T_{p} \mathcal{G}_{k}\right)=0\right\}$, is a submanifold of $\mathcal{N}_{k}$ transverse to $\mathcal{G}_{k}$.

A complete system of foliations on $\Sigma_{f}$ is a system of foliations on the union of all the strata constituting $\Sigma_{f}$.

Remark 5.7. Notice that the set $S_{1}, \ldots, S_{l}$ of $l$ smallest strata, arranged in decreasing order, satisfies the property that $S_{k} \cup \ldots \cup S_{l}$ is closed for all $1 \leq k \leq l$.

Remark 5.8. Given a system of foliations on $\cup_{k=1}^{l} S_{k}$, we will always assume (and this is not restrictive) that another system of tubular neighborhoods $\underline{\mathcal{N}}_{k}$ of open subsets $\underline{O}_{k}=\underline{\mathcal{N}}_{k} \cap S_{k}$ of $S_{k}$ as been given that satisfies $\overline{\mathcal{N}}_{k} \subset \mathcal{N}_{k}$ for all $k$, as well as property $a$ ) in Definition 5.6. Hence, the collection $\left\{\left.\mathcal{G}_{k}\right|_{\mathcal{N}_{k}}\right\}$ is also a system of foliations on $\cup_{k=1}^{l} S_{k}$. We will also use $\mathcal{N}_{k}^{\prime}, \mathcal{N}_{k}^{\prime \prime}, \ldots, \mathcal{N}_{k}^{i}{ }_{k}^{\prime}, \ldots$ to denote more tubular neighborhoods of open subsets $O_{k}^{\prime}, O_{k}^{\prime \prime}, \ldots, O_{k}^{i \prime}, \ldots$ of $S_{k}$ such that

$$
\overline{\mathcal{N}_{k}} \subset \mathcal{N}_{k}^{i \prime} \subset \overline{\mathcal{N}_{k}^{i \prime}} \subset \mathcal{N}_{k}^{(i-1) \prime} \subset \mathcal{N}_{k} .
$$

The need for these additional neighborhoods will appear in the proof of Proposition 5.9. It explains why, in Definition 5.6, we consider a tubular neighborhood of an open subset of a stratum, rather than a tubular neighborhood of the entire stratum. Indeed, if $\mathcal{N}$ is a tubular neighborhood of a nonclosed embedded submanifold $S$ (like most strata), there is no tubular neighborhood $\mathcal{N}^{\prime}$ of $S$ with $\overline{\mathcal{N}}^{\prime} \subset \mathcal{N}$. To obtain such an inclusion we have to replace $S$ by a submanifold $O$ of $S$ with $\bar{O} \subset S$.

Proposition 5.9 (Recurrence step). Let $S_{1}, \ldots, S_{l}$ be a set of strata satisfying the property that for all $k$, the set $S_{k} \cup \ldots \cup S_{l}$ is closed in $M$, and suppose that $\left\{\mathcal{G}_{k} ; k=1, \ldots, l\right\}$ is a system of foliations on $\cup_{k} S_{k}$. Let $S$ be another stratum for which $S \cup S_{1} \cup \ldots \cup S_{l}$ is closed. Then we can extend $\left\{\mathcal{G}_{k} ; k=1, \ldots, l\right\}$ to a system of foliations on $S \cup S_{1} \cup \ldots \cup S_{l}$.

The word extend has to be given the following meaning. A foliation $\mathcal{G}$ will be constructed on some tubular neighborhood $\mathcal{N}$ of an open subset $O$ of $S$ in such a way that $\mathcal{G}$ together with the restrictions of the foliations $\mathcal{G}_{k}$ to the neighborhoods $\underline{\mathcal{N}}_{k}$ is a system of foliations on $S \cup S_{1} \cup \ldots \cup S_{l}$. 
Proof. We will assume for our convenience that $\bar{S} \cap S_{k} \neq \emptyset$ for all $1 \leq k \leq l$ (if this is not true, select the $S_{k}$ 's intersecting $\bar{S}$ nontrivially, label them $S_{1}, \ldots, S_{l}$, and ignore the other ones until further notice). We can also assume that $\operatorname{dim} \mathcal{G}_{k} \geq \operatorname{dim} \mathcal{G}_{k+1}$ for all $1 \leq k \leq l-1$ (without affecting the property that $S_{k} \cup \ldots \cup S_{l}$ is closed for all $k$ ).

The proof follows the first three steps of Subsection 5.2. The difficulty lies in the second and third steps. We will need to adjust the distribution $\mathcal{D}^{\prime}$ and then the foliation $\mathcal{G}$ so as to make them match the foliations $\mathcal{G}_{k}$ already constructed.

Adjustment of $\mathcal{D}^{\prime}$. Suppose $S \subset \Sigma_{d_{o}}^{\left(i_{1}, \ldots, i_{k}\right)}$, in particular $\operatorname{rk} V_{S}^{+}=d_{o}$. We begin with describing a procedure that allows one to canonically extend a $d$-dimensional subspace of $T_{s} \mathcal{F}, s \in S$, on which $\bar{d}^{2} f$ is positive definite $(d$ may therefore not be greater than $d_{o}$ ), to a $d_{o}$-dimensional subspace with the same property.

Because $\bar{d}^{2} f$ induces a metric on $T_{S} \mathcal{F}$ whose rank and signature are constant, it yields a bundle decomposition $T_{S} \mathcal{F}=V^{+} \oplus V^{-} \oplus V^{0}$ into $g$ orthogonal and $\bar{d}^{2} f$-orthogonal subspaces (cf. beginning of Section 5). Let $p_{1}$ denote the projection $T_{S} \mathcal{F} \rightarrow V^{+}$, and let $p_{2}$ denote the projection $T_{S} \mathcal{F} \rightarrow$ $V^{-} \oplus V^{0}$. Given an element $X$ in $T_{S} \mathcal{F}$, write $X=X^{+}+X^{-}+X^{0}$, where $X^{+}, X^{-}$and $X^{0}$ belong to $V^{+}, V^{-}$and $V^{0}$ respectively. For $1 \leq d \leq d_{o}$, consider

$$
\begin{aligned}
\mathcal{W}^{+} & =\cup_{s \in S}\left(\mathcal{W}_{s}^{+}=\left\{X \in T_{s} \mathcal{F} ; \bar{d}^{2} f(X, X)>0\right\}\right) \\
\mathcal{P}^{+, d} & =\cup_{s \in S}\left(\mathcal{P}_{s}^{+, d}=\left\{P \in G^{d}\left(T_{s} \mathcal{F}\right) ; P-\{0\} \subset \mathcal{W}_{s}^{+}\right\}\right)
\end{aligned}
$$

where $G^{d}\left(T_{s} \mathcal{F}\right)$ denotes the Grassmann manifold of $d$-planes in $T_{s} \mathcal{F}$. If $E$ is a vector space endowed with an inner product, let $S(E)$ denote the unit sphere in $E$, and let $B(E)$ denote the open unit ball in $E$. Observe that if $P$ is a plane in $\mathcal{P}^{+, d}$ then $\left.p_{1}\right|_{P}$ is injective. Hence, an element $P$ of $\mathcal{P}_{s}^{+, d}$ is the graph of a linear map defined on $p_{1}(P)$ :

$$
\begin{aligned}
\varphi_{P}: p_{1}(P) & \rightarrow V_{s}^{-} \times V_{s}^{0} \\
x & \mapsto p_{2} \circ\left(\left.p_{1}\right|_{P}\right)^{-1}(x),
\end{aligned}
$$

whose restriction to the sphere $S\left(p_{1}(P)\right)$ takes its values in $B\left(V_{s}^{-}\right) \times V_{s}^{0}$. Conversely, an element of $G^{d}\left(T_{s} \mathcal{F}\right)$ that coincides with the graph of such a linear map belongs to $\mathcal{P}_{s}^{+, d}$. Notice that if $P$ is $d_{o}$-dimensional, then $p_{1}(P)=V_{s}^{+}$. 
Now given an element $P$ in some $\mathcal{P}_{s}^{+, d}$ with $d<d_{o}$, we extend it to an element $\mathcal{E}(P)$ in $\mathcal{P}_{s}^{+, d_{o}}$ as described hereafter. Let $q_{P}: V_{s}^{+} \rightarrow p_{1}(P)$ denote the orthogonal projection onto $p_{1}(P)$. Then define $\mathcal{E}(P)$ via its associated linear map $\varphi_{\mathcal{E}(P)}$ by:

$$
\begin{aligned}
\varphi_{\mathcal{E}(P)}: V_{s}^{+} & \rightarrow V_{s}^{-} \times V_{s}^{0} \\
x & \mapsto\left(\varphi_{P} \circ q_{P}\right)(x) .
\end{aligned}
$$

Remark 5.10. We can form convex linear combinations of elements in $\mathcal{P}^{+, d_{0}}$. Indeed, let $P_{1}, \ldots, P_{r}$ be elements of $\mathcal{P}_{s}^{+, d_{0}}$ for some $s$ in $S$, and let $a_{1}, \ldots, a_{r}$ be positive real numbers with $a_{1}+\ldots+a_{r}=1$. Then $a_{1} P_{1}+\ldots+a_{r} P_{r}$ denotes the element of $\mathcal{P}_{s}^{+, d_{0}}$ whose associated function $\varphi$ is $a_{1} \varphi_{P_{1}}+\ldots+a_{r} \varphi_{P_{r}}$. The map $\varphi$ takes its values in $B\left(V_{s}^{-}\right) \times V_{s}^{0}$, as this set is convex.

As in the first step of Section 5.2, the stratum $S$ is extended to a $q$ dimensional embedded submanifold $S^{\prime}$ transverse to $\mathcal{F}$. The manifold $S^{\prime}$ is the image of a fiberwise convex neighborhood $\mathcal{O}$ of the 0 -section in the bundle $p: E=\left(T S+T_{S} \mathcal{F}\right)^{\perp} \rightarrow S$ by the exponential map associated to $g$. Recall from the second step of Section 5.2, that a linear connection on $T \mathcal{F}$ determines, for each point $x$ in $S^{\prime}$, a linear isomorphism $i_{x}: T_{x_{o}} \mathcal{F} \rightarrow T_{x} \mathcal{F}$, where $x_{0}=p\left(\exp ^{-1}(x)\right)$. Set $\mathcal{O}_{k}^{\prime}=\exp \left(\mathcal{O} \cap p^{-1}\left(\mathcal{N}_{k}^{\prime} \cap S\right)\right)$ and $\mathcal{O}_{k}^{\prime \prime}=$ $\exp \left(\mathcal{O} \cap p^{-1}\left(\mathcal{N}_{k}^{\prime \prime} \cap S\right)\right)$.

Lemma 5.11. Provided $\mathcal{O}$ is sufficiently small, the following properties hold for all $k$ :

- $\mathcal{O}_{k}^{\prime} \subset \mathcal{N}_{k}$

- $\mathcal{O}_{k}^{\prime \prime} \supset \mathcal{N}_{k}^{\prime \prime \prime} \cap S^{\prime}$

- for every $x$ in $\mathcal{O}_{k}^{\prime}$, the space $i_{x}^{-1}\left(T_{x} \mathcal{G}_{k}\right)$ is contained in $\mathcal{W}_{x_{o}}^{+} \cup\{0\}$.

Proof. Given a subset $U$ of $S$ and a positive number $\varepsilon$, let $U^{\varepsilon}$ denote the subset $\exp \left(\cup_{s \in U} B_{\varepsilon}\left(0_{s}\right)\right)$ of $S^{\prime}$, where $B_{\varepsilon}\left(0_{s}\right)$ is the ball of radius $\varepsilon$ centered at 0 in $p^{-1}(s)$. For every $s$ in $S$, there exists a neighborhood $U_{s}$ of $s$ in $S$ and a positive number $\varepsilon_{s}$ such that

- $U_{s}^{\varepsilon_{s}} \subset \mathcal{N}_{k}$ for all $k$ for which $s \in \overline{\mathcal{N}}_{k}^{\prime}$,

- $U_{s} \cap \mathcal{N}_{k}^{\prime}=\emptyset$ for all $k$ for which $s \notin \overline{\mathcal{N}}_{k}^{\prime}$, 
- $U_{s} \subset \mathcal{N}_{k}^{\prime \prime}$ for all $k$ for which $s \in \overline{\mathcal{N}}_{k}^{\prime \prime \prime}$,

- $U_{s}^{\varepsilon_{s}} \cap \mathcal{N}_{k}^{\prime \prime \prime}=\emptyset$ for all $k$ for which $s \notin \overline{\mathcal{N}}_{k}^{\prime \prime \prime}$,

- $i_{x}^{-1}\left(T_{x} \mathcal{G}_{k}\right) \subset \mathcal{W}_{x_{0}}^{+} \cup\{0\}$ for all $x \in U_{s}^{\varepsilon_{s}}$ with $s$ in $\overline{\mathcal{N}}_{k}^{\prime}$.

Let $\left\{U_{i} ; i \geq 1\right\}$ be a locally finite refinement of the covering of $S$ by the $U_{s}^{\varepsilon_{s}}$ 's. For any fiberwise convex neighborhood $\mathcal{O}$ of the 0 -section in $E$ such that $\exp (\mathcal{O}) \subset \cup_{i} U_{i}$, the required three properties are satisfied.

For all $k=1, \ldots, l$, the dimension of $\mathcal{G}_{k}$ is at most equal to $d_{o}$ (we supposed that $\bar{S} \cap S_{k} \neq \emptyset$ for all $k$, and this implies that $\operatorname{rk} V_{S}^{+} \geq \operatorname{rk} V_{S_{k}}^{+}$ for all $k$ ). We can therefore associate to each foliation $\mathcal{G}_{k}$ a $d_{o}$-dimensional distribution $\mathcal{D}_{k}^{\prime}$, defined on $\mathcal{O}_{k}^{\prime}$, tangent to $\mathcal{F}$, and containing $T \mathcal{G}_{k}$. Simply let

$$
\left(\mathcal{D}_{k}^{\prime}\right)_{x}=i_{x}\left(\mathcal{E}\left(i_{x}^{-1}\left(T_{x} \mathcal{G}_{k}\right)\right)\right) \text {. }
$$

We would like to paste the $\mathcal{D}_{k}^{\prime}$ 's together so as to obtain a $d_{o^{-}}$-dimensional distribution $\mathcal{D}^{\prime} \subset T_{S^{\prime}} \mathcal{F}$ on $S^{\prime}$ containing the distributions $T_{S^{\prime} \cap \mathcal{N}_{k}^{\prime \prime \prime}} \mathcal{G}_{k}$, and for which $\bar{d}^{2} f$ is positive definite on $\mathcal{D}=\left.\mathcal{D}^{\prime}\right|_{S}$. For each $k$ between 1 and $l$, let $\rho_{k}: M \rightarrow[0,1]$ be a smooth function equal to 1 near $\mathcal{N}_{k}^{\prime \prime}$ and vanishing near the complement of $\mathcal{N}_{k}^{\prime}$. We define $\mathcal{D}^{\prime}$ as follows:

$$
\begin{aligned}
\mathcal{D}_{x}^{\prime}= & \rho_{1}\left(x_{o}\right)\left(\mathcal{D}_{1}^{\prime}\right)_{x}+\left(1-\rho_{1}\left(x_{o}\right)\right) \rho_{2}\left(x_{o}\right)\left(\mathcal{D}_{2}^{\prime}\right)_{x}+\ldots+ \\
& \left(1-\rho_{1}\left(x_{o}\right)\right) \ldots\left(1-\rho_{k-1}\left(x_{o}\right)\right) \rho_{k}\left(x_{o}\right)\left(\mathcal{D}_{k}^{\prime}\right)_{x}+\ldots+ \\
& \left(1-\rho_{1}\left(x_{o}\right)\right) \ldots\left(1-\rho_{l}\left(x_{o}\right)\right) i_{x}\left(V_{x_{o}}^{+}\right)
\end{aligned}
$$

where $x$ is in $S^{\prime}$, and where $x_{0}=p\left(\exp ^{-1}(x)\right)$. It is understood that when some distribution $\mathcal{D}_{k}^{\prime}$ is not defined at the point $x$, the quantity $\rho_{k}\left(x_{o}\right)\left(\mathcal{D}_{k}^{\prime}\right)_{x}$ is defined to be $\rho_{k}\left(x_{o}\right) i_{x}\left(V_{x_{o}}^{+}\right)$. The linear combination appearing in the right hand side has to be understood as follows. If $P_{1}, \ldots, P_{r}$ are subspaces of $T_{x} \mathcal{F}$ for some $x$ in $S^{\prime}$ such that $i_{x}^{-1}\left(P_{i}\right) \subset \mathcal{W}^{+} \cup\{0\}$, and if $a_{1}, \ldots, a_{r}$ are real numbers, then

$$
a_{1} P_{1}+\ldots+a_{r} P_{r}=i_{x}\left(a_{1} i_{x}^{-1}\left(P_{1}\right)+\ldots+a_{r} i_{x}^{-1}\left(P_{r}\right)\right),
$$

where the linear combination in the right hand side has to be interpreted according to Remark 5.10. Observe that on $\mathcal{O}_{k}^{\prime \prime}$, hence on $\mathcal{N}_{k}^{\prime \prime \prime} \cap S^{\prime}$, the distribution $\mathcal{D}^{\prime}$ contains $T \mathcal{G}_{k}$. Indeed, the function $\left(1-\rho_{k}\left(x_{o}\right)\right)$ vanishes on $\mathcal{N}_{k}^{\prime \prime}$, hence, only $\mathcal{D}_{1}^{\prime}, \ldots, \mathcal{D}_{k}^{\prime}$ are involved in the definition of $\mathcal{D}_{x}^{\prime}$ for $x$ in $\mathcal{O}_{k}^{\prime \prime}$. By construction, $\mathcal{D}_{j}^{\prime}$ contains $T \mathcal{G}_{k}$ for $j$ between 1 and $k$; hence, any convex linear combination of $\mathcal{D}_{1}^{\prime}, \ldots, \mathcal{D}_{k}^{\prime}$ contains $T \mathcal{G}_{k}$ as well. 
To keep notations light, we denote $\mathcal{N}_{k}^{\prime \prime \prime}$ by $\mathcal{N}_{k}, \mathcal{N}_{k}^{\prime \prime \prime \prime}$ by $\mathcal{N}_{k}^{\prime}$, and so forth, while fixing $\underline{\mathcal{N}}_{k}$.

Adjustment of $\mathcal{G}$. We will now extend the distribution $\mathcal{D}^{\prime}$ to a foliation $\mathcal{G} \subset \mathcal{F}$ defined on a tubular neighborhood $\mathcal{N}$ of $S^{\prime}$, that contains $\mathcal{G}_{k}$ on $\mathcal{N} \cap \mathcal{N}_{k}^{\prime \prime \prime}$. Recall from the previous section the foliated exponential map:

$$
\xi: \mathcal{U} \subset T_{S^{\prime}} \mathcal{F} \rightarrow M
$$

where the set $\mathcal{U}$ is a fiberwise convex neighborhood of the 0 -section in $T_{S^{\prime}} \mathcal{F}$, small enough for $\xi$ to be an embedding. Let $\tilde{\mathcal{G}}_{k}$ denote the foliation $(\xi)_{*}^{-1}\left(\mathcal{G}_{k}\right)$ defined on $\xi^{-1}\left(\mathcal{N}_{k}\right)$. It is of course tangent to the foliation of $\mathcal{U}$ by the fibers of the natural projection $\pi: T_{S^{\prime}} \mathcal{F} \rightarrow S^{\prime}$. For all $k=1, \ldots, l$, let $\mathcal{E}_{k}=$ $\left(T \mathcal{G}_{k}\right)^{\perp} \cap T \mathcal{F}$. Given any $k$, there exists a (not necessarily fiberwise convex) neighborhood $\mathcal{V}_{k}$ of the 0-section of $T_{S^{\prime} \cap \mathcal{N}_{k}} \mathcal{F}$ contained in $\xi^{-1}\left(\mathcal{N}_{k}\right)$, such that each leaf of $\tilde{\mathcal{G}}_{k} \mid \mathcal{V}_{k}$ intersects $\mathcal{E}_{k}$ along exactly one point. In particular, there is a map $g_{k}: \mathcal{V}_{k} \rightarrow \mathcal{E}_{k}$ defined by $\left\{g_{k}(x)\right\}=\left(\tilde{G}_{k}\right)_{x} \cap \mathcal{E}_{k}$, where $\left(\tilde{G}_{k}\right)_{x}$ denotes the leaf of $\tilde{\mathcal{G}}_{k} \mid \mathcal{V}_{k}$ containing $x$. The following lemma is very similar to Lemma 5.11 although it is formulated in $T_{S^{\prime}} \mathcal{F}$ instead of $M$. Its proof is omitted. Let $\mathcal{U}_{k}^{\prime}$ (respectively $\left.\mathcal{U}_{k}^{\prime \prime}\right)$ denote the set $\mathcal{U} \cap \pi^{-1}\left(\mathcal{N}_{k}^{\prime} \cap S^{\prime}\right)$ (respectively $\mathcal{U} \cap \pi^{-1}\left(\mathcal{N}_{k}^{\prime \prime} \cap S^{\prime}\right)$ ).

Lemma 5.12. Provided $\mathcal{U}$ is sufficiently small, the following properties hold for all $k$.

$$
\begin{aligned}
& \text { - } \mathcal{U}_{k}^{\prime} \subset \mathcal{V}_{k}, \\
& \text { - } \mathcal{U}_{k}^{\prime \prime} \supset \xi^{-1}\left(\mathcal{N}_{k}^{\prime \prime \prime}\right) .
\end{aligned}
$$

Let $g_{l+1}$ denote the orthogonal projection $T_{S^{\prime}} \mathcal{F} \rightarrow \mathcal{D}^{\prime \perp}$, and let $\nu$ : $T_{S^{\prime}} \mathcal{F} \times T_{S^{\prime}} \mathcal{F} \rightarrow T_{S^{\prime}} \mathcal{F}$ be the map that sends the pair $(x, y)$ to the orthogonal projection of $x$ onto the coset of $\mathcal{D}^{\prime}$ passing through the point $y$ (that is, $\left.\nu(x, y)=x+g_{l+1}(y-x)\right)$. For each $1 \leq k \leq l$, consider a smooth function $\rho_{k}: M \rightarrow[0,1]$ whose value is 1 near $\mathcal{N}_{k}^{\prime \prime}$, and is 0 near the complement of $\mathcal{N}_{k}^{\prime}$. Define $\mu: \mathcal{U} \rightarrow T_{S^{\prime}} \mathcal{F}$ by

$$
\begin{aligned}
\mu(x)= & \nu\left(x, \rho_{1}\left(x_{o}\right) g_{1}(x)+\left(1-\rho_{1}\left(x_{o}\right)\right) \rho_{2}\left(x_{o}\right) g_{2}(x)+\ldots+\right. \\
& \left(1-\rho_{1}\left(x_{o}\right)\right) \ldots\left(1-\rho_{k-1}\left(x_{o}\right)\right) \rho_{k}\left(x_{o}\right) g_{k}(x)+\ldots+ \\
& \left.\left(1-\rho_{1}\left(x_{o}\right)\right) \ldots\left(1-\rho_{l}\left(x_{o}\right)\right) g_{l+1}(x)\right)
\end{aligned}
$$

where $x_{o}=\pi(x)$, and where, if $g_{k}(x)$ is not defined, we set $\rho_{k}\left(x_{o}\right) g_{k}(x)=0_{x_{o}}$. Observe that the map $\mu$ coincides with the identity on the 0 -section. Denote by $\mathcal{G}^{\prime}$ the foliation of $T_{S^{\prime}} \mathcal{F}$ by the cosets of $\mathcal{D}^{\prime}$. The idea is that, provided $\mu$ is a diffeomorphism, the foliation $\mu\left(\left.\tilde{\mathcal{G}}_{k}\right|_{\mathcal{U}_{k}^{\prime \prime}}\right)$ is tangent to the foliation $\mathcal{G}^{\prime}$. 
Lemma 5.13. There exists a fiberwise convex neighborhood $\tilde{\mathcal{U}} \subset \mathcal{U}$ of the 0 -section in $T_{S^{\prime}} \mathcal{F}$ for which $\mu: \tilde{\mathcal{U}} \rightarrow T_{S^{\prime}} \mathcal{F}$ is a diffeomorphism onto its image, another neighborhood of the 0 -section in $T_{S^{\prime}} \mathcal{F}$.

Proof. First observe that the map $\mu_{k}(x)=\nu\left(x, g_{k}(x)\right)$, defined for $x$ in $\mathcal{V}_{k}$, coincides with the identity on $\mathcal{V}_{k} \cap \mathcal{E}_{k}$ (or on $T_{S^{\prime}} \mathcal{F}$ for $k=l+1$ ), and that $\left(\mu_{k}\right)_{*}(X)=X$ for $X$ in $T_{s} \mathcal{G}_{k}$, with $s$ in $S^{\prime} \cap \mathcal{V}_{k}$ (as a consequence of the fact that $\left(\mathcal{D}^{\prime}\right)_{s}$ contains $T_{s} \mathcal{G}_{k}$ for $s$ in $\left.S^{\prime} \cap \mathcal{N}_{k}\right)$. Hence, the map $\left(\mu_{k}\right)_{*_{s}}$ coincides with the identity map for $s$ in $S^{\prime} \cap \mathcal{V}_{k}$. Observe now that, if we fix $s$ in $S^{\prime}$ and let $x$ vary in $T_{s} \mathcal{F} \cap \mathcal{U}$, the map $\mu$ can be written as :

$$
\mu(x)=\nu\left(x, t_{1} g_{1}(x)+\ldots+t_{l} g_{l}(x)+t_{l+1} g_{l+1}(x)\right),
$$

where $t_{k}=\left(1-\rho_{1}(s)\right) \ldots\left(1-\rho_{k-1}(s)\right) \rho_{k}(s)$ for $1 \leq k \leq l$, and where $t_{l+1}=\left(1-\rho_{1}(s)\right) \ldots\left(1-\rho_{l}(s)\right)$. Hence, for $X$ in $T_{0_{s}} T_{s} \mathcal{F} \simeq T_{s} \mathcal{F}$, we have

$$
\begin{aligned}
\mu_{*_{s}}(X) & =\nu_{*_{(s, s)}}\left(X,\left(t_{1} g_{1}+\ldots+t_{l+1} g_{l+1}\right)_{*_{s}}(X)\right) \\
& =\nu_{*_{(s, s)}}\left(\left(t_{1}+\ldots+t_{l+1}\right) X, t_{1}\left(g_{1}\right)_{*_{s}}(X)+\ldots+t_{l+1}\left(g_{l+1}\right)_{*_{s}}(X)\right) \\
& =t_{1} \nu_{*_{(s, s)}}\left(X,\left(g_{1}\right)_{*_{s}}(X)\right)+\ldots+t_{l+1} \nu_{*_{(s, s)}}\left(X,\left(g_{l+1}\right)_{*_{s}}(X)\right) \\
& =t_{1}\left(\mu_{1}\right)_{*_{s}}(X)+\ldots+t_{l+1}\left(\mu_{l+1}\right)_{*_{s}}(X) \\
& =t_{1} X+\ldots+t_{l+1} X \\
& =X
\end{aligned}
$$

Thus $\mu_{*_{s}}=$ Id for all $s$ in $S^{\prime}$, and the lemma follows.

Lemma 5.14. For all $k$, the foliation $\mu_{*}^{-1}\left(\mathcal{G}^{\prime}\right)$ contains the foliation $\tilde{\mathcal{G}}_{k}$ on $\mathcal{U}_{k}^{\prime \prime} \cap \tilde{\mathcal{U}}$, hence on $\xi^{-1}\left(\mathcal{N}_{k}^{\prime \prime \prime}\right) \cap \tilde{\mathcal{U}}$.

Proof. If $x$ belongs to $\mathcal{U}_{k}^{\prime \prime} \cap \tilde{\mathcal{U}}$, then $\left(1-\rho_{k}\left(x_{o}\right)\right)=0$. Hence the map $\mu$ can be expressed as follows:

$$
\begin{aligned}
\mu(x)= & \nu\left(x, \rho_{1}\left(x_{o}\right) g_{1}(x)+\left(1-\rho_{1}\left(x_{o}\right)\right) \rho_{2}\left(x_{o}\right) g_{2}(x)+\ldots+\right. \\
& \left.\left(1-\rho_{1}\left(x_{o}\right)\right) \ldots\left(1-\rho_{k-1}\left(x_{o}\right)\right) \rho_{k}\left(x_{o}\right) g_{k}(x)\right) .
\end{aligned}
$$

Besides, for all $j=1, \ldots, k$, the map $x \mapsto \rho_{j}\left(x_{o}\right) g_{j}(x)$ is constant on the leaf $\left(\tilde{G}_{k}\right)_{x}$ of $\left.\tilde{\mathcal{G}}_{k}\right|_{\mathcal{U}_{k}^{\prime \prime} \cap \tilde{\mathcal{U}}}$ passing through $x$. This assertion follows from the fact that whenever $k_{1}>k_{2}$, the distribution $T \mathcal{G}_{k_{1}}$ contains the distribution $T \mathcal{G}_{k_{2}}$ on $\mathcal{N}_{k_{1}} \cap \mathcal{N}_{k_{2}}$. Thus the map $\mu$ sends the entire leaf $\left(\tilde{G}_{k}\right)_{x}$ into a coset of $\mathcal{D}^{\prime}$. 
The foliation $\mathcal{G}$ is defined to be $\xi_{*}\left(\left.\mu_{*}^{-1}\left(\mathcal{G}^{\prime}\right)\right|_{\tilde{\mathcal{U}}}\right)$. Its domain is the open set $\mathcal{N}=\xi(\tilde{\mathcal{U}})$. Lemma 5.14 implies that $\mathcal{G}$ contains $\mathcal{G}_{k}$ on $\mathcal{N}_{k}^{\prime \prime \prime} \cap \mathcal{N}$. Define $P$ to be the foliated singular locus of $f$ with respect to $\mathcal{G}$, that is, $P=$ $\left\{p \in \mathcal{N} ; d f(p)\left(T_{p} \mathcal{G}\right)\right\}$. We may assume, after shrinking $\mathcal{N}$ if needed, that $\bar{d}^{2} f$ is positive definite on $T_{P} \mathcal{G}$ (cf. Fourth step of Subsection 5.2). The strata we might have ignored in the very beginning of this proof should now be re-incorporated.

Lemma 5.15. The foliations $\mathcal{G}, \mathcal{G}_{1}, \ldots, \mathcal{G}_{l}$, defined on $\mathcal{N}, \underline{\mathcal{N}}_{1}, \ldots, \underline{\mathcal{N}}_{l}$ respectively, form a system of foliations on $S \cup S_{1} \cup \ldots \cup S_{l}$.

Proof. Among the five defining properties of a system of foliations, properties a), c), d) and e) have been taken care of during the construction. Only b) requires some attention. We need to make sure that $\mathcal{N}$ does not intersect the tubular neighborhoods of the strata that we discarded at the beginning

of the proof. A way to insure this is to fix a family of neighborhoods $\tilde{\mathcal{N}}_{k}$, one for each strata $S_{k}$, satisfying the following property:

$$
\text { if } \bar{S}_{k_{1}} \cap S_{k_{2}}=\emptyset=S_{k_{1}} \cap \bar{S}_{k_{2}} \text {, then } \tilde{\mathcal{N}}_{k_{1}} \cap \tilde{\mathcal{N}}_{k_{2}}=\emptyset \text { as well. }
$$

Then, whenever we consider a neighborhood of some strata $S_{k}$, we request that it be contained in $\tilde{\mathcal{N}}_{k}$.

End of the proof of Proposition 5.9.

Corollary 5.16. A complete system of foliations on $\Sigma_{f}$ exists.

Proof. Let $S_{1}, \ldots, S_{l}$ be the set of all strata of $\Sigma_{f}$, presented in such a way that $S_{1}>S_{2}>\ldots$ Then, as described in Subsection 5.2 (or in Proposition 5.9 with $l=0$ ), we can construct a foliation $\mathcal{G}_{l}$ near $S_{l}$ and use Proposition 5.9 repeatedly to extend $\mathcal{G}_{l}$ to a system of foliations near $\cup_{k} S_{k}$.

\subsection{Construction of a metric from a complete system of}

\section{foliations.}

Let $\left\{\mathcal{G}_{k} ; k=1, \ldots, l\right\}$ be a complete system of foliations on $\Sigma_{f}$. Each $\mathcal{G}_{k}$ is defined on a tubular neighborhood $\mathcal{N}_{k}$ of an open subset $O_{k}$ of the stratum $S_{k}$. The foliated singular locus of $f$ with respect to $\mathcal{G}_{k}$ is denoted by $P_{k}$. We suppose that $\operatorname{dim} \mathcal{G}_{k} \geq \operatorname{dim} \mathcal{G}_{k+1}$ for all $k$. 
Definition 5.17. A Riemannian metric is said to be adapted to the system of foliations $\left\{\mathcal{G}_{k} ; k=1, \ldots, l\right\}$ if $T_{x} P_{k}$ is perpendicular to $T_{x} \mathcal{G}_{k}$ for all $x$ in $P_{k} \cap \mathcal{N}_{k}^{\prime}$.

Proposition 5.18. A Riemannian metric adapted to the system of foliations $\left\{\mathcal{G}_{k} ; k=1, \ldots, l\right\}$ exists.

Proof. For $x$ in $M$, let $U_{x}$ be a neighborhood of $x$ in $M$, and let $h_{x}$ be a Riemannian metric defined on $U_{x}$ such that

1. $U_{x} \subset \mathcal{N}_{k}$ for all $k$ for which $x \in P_{k} \cap \overline{\mathcal{N}}_{k}^{\prime}$,

2. $U_{x} \cap P_{k} \cap \overline{\mathcal{N}}_{k}^{\prime}=\emptyset$ for all $k$ for which $x \notin P_{k} \cap \overline{\mathcal{N}}_{k}^{\prime}$,

3. if $x \in P_{k} \cap \overline{\mathcal{N}}_{k}^{\prime}$, then $T_{y} P_{k} \perp_{h_{x}} T_{y} \mathcal{G}_{k}$ for all $y$ in $U_{x} \cap P_{k}$.

Existence of $U_{x}$ and $h_{x}$ is easily seen, except perhaps for the last condition. Suppose that $x$ belongs to $\overline{\mathcal{N}}_{k}^{\prime} \cap P_{k}$ if and only if $k \in\left\{k_{1}, k_{2}, \ldots, k_{r}\right\}$, with $k_{1}<k_{2}<\ldots<k_{r}$. Then for Property 3 . to hold, it is sufficient that the following bundles be pairwise $h_{x}$-orthogonal.

$$
T P_{k_{1}}, T P_{k_{2}} \cap T \mathcal{G}_{k_{1}}, T P_{k_{3}} \cap T \mathcal{G}_{k_{2}}, \ldots, T P_{k_{r}} \cap T \mathcal{G}_{k_{r-1}}, T \mathcal{G}_{k_{r}} .
$$

These bundles span $T M$ on $U_{x} \cap\left(\cap_{j}\left(\overline{\mathcal{N}}_{k_{j}}^{\prime} \cap P_{k_{j}}\right)\right)$ and are linearly independent, so that the Gram-Schmidt orthogonalization process can be carried out.

let $\left\{U_{i} ; i \geq 1\right\}$ be a locally finite refinement of the covering of $M$ by the $U_{x}$ 's, and let $\left\{\theta_{i}\right\}$ be a partition of unity subordinate to the covering $\left\{U_{i}\right\}$. Denote by $h_{i}$ the metric $h_{x_{i}}$, where $x_{i}$ is chosen in such a way that $U_{i} \subset U_{x_{i}}$. Define

$$
h=\sum_{i \geq 1} \theta_{i} h_{i}
$$

Then $T_{y} P_{k} \perp_{h} T_{y} \mathcal{G}_{k}$ whenever $y$ belongs to $P_{k} \cap \mathcal{N}_{k}^{\prime}$. Indeed, let $y \in P_{k} \cap \mathcal{N}_{k}^{\prime}$, and suppose that $y$ belongs to $U_{i} \subset U_{x_{i}}$ for some $i$. Then $x_{i} \in P_{k} \cap \overline{\mathcal{N}}_{k}^{\prime}$. Hence $T_{y} P_{k}$ is perpendicular to $T_{y} \mathcal{G}_{k}$ with respect to the metric $h_{i}$. Since this holds true for every $i$ for which $y \in U_{i}$, we have $T_{y} P_{k} \perp_{h} T_{y} \mathcal{G}_{k}$.

\subsection{Conclusion.}

The preceding subsections show how to construct a complete system of foliations $\left\{\mathcal{G}_{k} ; k=1, \ldots, l\right\}$ on $\Sigma_{f}$ (Definition 5.6 and Corollary 5.16), as well as 
a Riemannian metric $h$ adapted to that system (Definition 5.17 and Proposition 5.18). By Proposition 5.3, there exists, for every $k$, a neighborhood $\mathcal{M}_{k}$ of $\mathcal{N}_{k}^{\prime} \cap \Sigma_{f}$ for which

$$
\mathcal{W}_{\mathcal{M}_{k}}\left(\Sigma_{f}\right) \subset P_{k}
$$

where $P_{k}$ denotes the foliated singular locus of $f$ with respect to the foliation $\mathcal{G}_{k}$. The submanifold $P_{k}$ has codimension at least one and intersect $\mathcal{F}$ transversely. Notice that the $\mathcal{M}_{k}$ 's cover $\Sigma_{f}$.

\section{The proof.}

Let us recall the statement of Theorem 0.4 whose proof will be completed in the present section.

Theorem 6.1. On a uniformely open foliated manifold, any open relation invariant under foliated isotopies satisfies the parametric h-principle.

Proof. Let $\Omega$ be an open relation invariant under foliated isotopies on the uniformely open foliated manifold $(M, \mathcal{F})$. Consider a proper $\mathcal{F}$-generic function $f: M \rightarrow[0, \infty)$ without leafwise local maxima. As observed in Remark 3.24, we may assume, without loss of generality, that $f$ is not only $\mathcal{F}$-generic but also strongly $\mathcal{F}$-generic. Let $\Sigma_{f}=S_{1} \cup \ldots \cup S_{l}$ be the decomposition of the foliated singular locus of $f$ (Definition 3.2) into strata (Definition 5.2). Let $\left\{\mathcal{G}_{k} ; k=1, \ldots, l\right\}$ be a complete system of foliations (Definition 5.6 and Corollary 5.16), endowed with an adapted metric $h$ (Definition 5.17 and Proposition 5.18). As before, we will denote by $\mathcal{N}_{k}$ the domain of definition of the foliation $\mathcal{G}_{k}$, by $P_{k}$ the foliated singular locus of $f$ with respect to the foliation $\mathcal{G}_{k}$, and by $\mathcal{M}_{k}$ a neighborhood of $\mathcal{N}_{k} \cap \Sigma_{f}$ for which $\mathcal{W}_{\mathcal{M}_{k}}\left(\Sigma_{f}\right) \subset P_{k}$ (Proposition 5.3). The leafwise gradient vector field of $f$ with respect to $h$ (Definition 4.1) is denoted as before by $\nabla_{\mathcal{F}} f$, and its local flow by $\varphi^{t}$. We will need a partition $\mathcal{P}=\left\{a_{0}=0=a_{1}<a_{2}<\ldots<a_{i}<\ldots\right\}$ of $[0, \infty)$ by non critical values (except for $a_{0}$ and $a_{1}$ ) of $f$. The latter provides us with an exhaustion $M=\cup_{i} K_{i}$ of $M$ by compact subsets $K_{i}=f^{-1}\left(\left[a_{i}, a_{i+1}\right]\right)$.

The proof of Theorem 6.1 consists in showing that, provided the partition $\mathcal{P}$ is fine enough, the hypotheses of Proposition 1.12 are satisfied. The first hypothesis, that the h-principle is valid near $K_{0}$ is easy to handle. Indeed, $K_{0}=f^{-1}(0)$ is a finite union of points (we assume here that the minimum 
value of $f$ is 0 ), and the local h-principle (Definition 1.8) is valid for any open relation (cf. Proposition 1.9), in particular for $\Omega$. Concerning the second hypothesis, that the h-principle for extensions is valid on each pair $\left(K_{i+1}, K_{i}\right)$, it is proven in two steps. The combination of Lemma 4.5 and Lemma 1.11 proves the h-principle for extensions on each pair $\left(K_{i+1}, K_{i} \cup\right.$ $\left.\mathcal{W}_{a_{i+1}}^{a_{i+2}}\left(\Sigma_{f}\right)\right)$. It remains to prove that the h-principle for extensions is valid on each pair $\left(K_{i} \cup \mathcal{W}_{a_{i+1}}^{a_{i+2}}\left(\Sigma_{f}\right), K_{i}\right)$. This is where the construction of a Riemannian metric carried out in Section 5 is needed, as will become clear below. Fix a slice $K_{i}$.

Observation 6.2. Since $f$ is a strongly $\mathcal{F}$-generic function on $(M, \mathcal{F})$, for each leaf $F$ of the foliation $\mathcal{F}$, the critical points of $\left.f\right|_{F}$ are isolated in $F$. Moreover, the submanifold $\Sigma_{f}$ is closed and embedded. Hence, for each $\sigma$ in $\Sigma_{f}$, there exists a neighborhood $U_{\sigma}$ of $\sigma$ in $M$ satisfying the following properties :

1. $U_{\sigma}$ is the domain of a chart $\left(U_{\sigma}, \varphi_{\sigma}\right)$ adapted to $\mathcal{F}$ and centered at $\sigma$ such that $\varphi_{\sigma}\left(U_{\sigma}\right)=B_{\sigma}^{1} \times B_{\sigma}^{2}$, where $B_{\sigma}^{1}$ is a closed ball about $\varphi(\sigma)$ in the image of the leaf $F_{\sigma}$, and where $B_{\sigma}^{2}$ is a closed ball about $\varphi(\sigma)$ in the transverse direction.

2. $U_{\sigma}$ is contained in $\mathcal{M}_{k}$ for some $k$.

3. The compact set $b U_{\sigma} \stackrel{\text { def. }}{=} \varphi_{\sigma}^{-1}\left(\partial B_{\sigma}^{1} \times B_{\sigma}^{2}\right)$ does not intersect $\Sigma_{f}$.

Let $\left\{U_{r} ; r=1,2, \ldots\right\}$ be a locally finite refinement of the cover of $\Sigma_{f}$ by the $U_{\sigma}$ 's. Observe that $\cup_{r} b U_{r}$ is a closed set, and that for only finitely many $r$ 's, the set $U_{r}$ intersects $K_{i}$ nontrivially. For each $r$, choose a $\sigma_{r}$ in $\Sigma_{f}$ such that $U_{r} \subset U_{\sigma_{r}}$. Choose also an index $k_{r} \in\{1, \ldots, l\}$ for which $U_{r} \subset \mathcal{M}_{k_{r}}$. We will use the notations introduced in Section 4.

\section{Lemma 6.3.}

$$
\mathcal{W}\left(\Sigma_{f}\right) \subset \bigcup_{r} \mathcal{S}\left(U_{r} \cap P_{k_{r}}\right)
$$

Proof. Let $x$ be a point in $M-\Sigma_{f}$ such that $x^{+}$is in $\Sigma_{f}$. The critical point $x^{+}$belongs to $U_{r}$ for some $r$. Either the trajectory $\varphi^{[0, \infty)}(x)$ intersects $b U_{r}$, or it is entirely contained in $U_{r}$. In the second case, we deduce from Proposition 5.3, and from the fact that $U_{r} \subset \mathcal{M}_{k_{r}}$, that $x$ must be contained in $U_{r} \cap P_{k_{r}}$. In the first case, let $x_{0}=\varphi^{t}(x)$ be the point in $\varphi^{[0, \infty)}(x) \cap b U_{r}$ for which $t$ is maximum. Then $\varphi^{[t, \infty)}(x)$ is entirely contained in $U_{r}$, and 
Proposition 5.3 implies that $x_{0}$ must belong to $P_{k_{r}}$. In both cases, $x$ belongs to $\mathcal{S}\left(U_{r} \cap P_{k_{r}}\right)$.

Lemma 6.4. Let

$$
\varepsilon_{i}=\inf \left\{f(x)-f\left(x^{-}\right) ; x \in \cup_{r}\left(b U_{r} \cap P_{k_{r}}\right) \cap K_{i} \text { and } x^{-} \in K_{i}\right\} .
$$

Then $\varepsilon_{i}$ is positive.

Proof. Suppose on the contrary that $\varepsilon_{i}=0$. Then there exists a sequence $\left(x_{j}\right)$ of points in $\cup_{r} b U_{r} \cap K_{i}$ with $x_{j}^{-}$in $K_{i}$ such that $\lim _{j \rightarrow \infty}\left(f\left(x_{j}\right)-f\left(x_{j}^{-}\right)\right)=$ 0 . We may assume, after extracting a subsequence if necessary, that the sequence $\left(x_{j}\right)$ converges in $K_{i}$ to a point $x$. Since $x$ belongs necessarily to $\cup_{r} b U_{r}$, it is not a leafwise critical point of $f$, and we may therefore consider a chart $(U, \psi)$ about $x$, adapted to the foliation $\mathcal{H}$ of $M-\Sigma_{f}$ by the orbits of $\nabla_{\mathcal{F}} f$. For all sufficiently large $j$, the point $x_{j}$ lies in $U$. Letting $x^{\prime}=\varphi^{t}(x)$ with $t<0$ be an element in $U$, there exists a sequence $x_{j}^{\prime}=\varphi^{t_{j}}\left(x_{j}\right)$ with $t_{j}<0$ converging to $x^{\prime}$. Because $f$ is strictly increasing along nonconstant trajectories of $\nabla_{\mathcal{F}} f$,

$$
\begin{aligned}
0 & <f(x)-f\left(x^{\prime}\right) \\
& =\lim _{j \rightarrow \infty}\left(f\left(x_{j}\right)-f\left(x_{j}^{\prime}\right)\right) \\
& \leq \lim _{j \rightarrow \infty}\left(f\left(x_{j}\right)-f\left(x_{j}^{-}\right)\right)
\end{aligned}
$$

contradicting the hypothesis that $\lim _{j \rightarrow \infty}\left(f\left(x_{j}\right)-f\left(x_{j}^{-}\right)\right)=0$.

Now let $\mathcal{P}^{\prime}=\left\{b_{0}=0=b_{1}<b_{2}<\ldots<b_{j} \ldots\right\}$ be a refinement of the partition $\mathcal{P}$ such that, if $a_{i}=b_{j_{i}}$, then $b_{j+1}-b_{j}<\varepsilon_{i}$, whenever $j_{i} \leq j<j_{i+1}$. Fix $j$, let $L_{j}$ denote the slice $f^{-1}\left(\left[b_{j}, b_{j+1}\right]\right)$, and let $i$ be the index for which $\left[b_{j}, b_{j+1}\right] \subset\left[a_{i}, a_{i+1}\right]$.

Lemma 6.5. For all $r$, the set $N_{r}=\mathcal{S}\left(U_{r} \cap P_{k_{r}} \cap L_{j}\right) \cap L_{j}$ is a finite union of compact subsets of embedded submanifolds of codimension at least one, transverse to $\mathcal{F}$.

Remark 6.6. It is not true in general that $N_{r}$ is an embedded submanifold. The problem is that, even though $\nabla_{\mathcal{F}} f$ is a leafwise gradient vector field, there might be trajectories leaving $U_{r} \cap P_{k_{r}}$ and coming back later to $U_{r} \cap P_{k_{r}}$, creating in the process self-intersections in $N_{r}$. 
Proof of Lemma 6.5. As in the proof of Lemma 6.4, let $\mathcal{H}$ denote the foliation of $M-\Sigma_{f}$ by the orbits of $\nabla_{\mathcal{F}} f$. For any point $x$ in $b U_{r} \cap P_{k_{r}} \cap L_{j}$, there exists a $t_{x} \leq 0$ for which $\varphi^{t_{x}}(x)=x_{0}$ belongs to $f^{-1}\left(b_{j}\right)-\Sigma_{f}$. Indeed, $b_{j+1}-b_{j}<\varepsilon_{i}$, but $f(x)-f\left(x^{-}\right) \geq \varepsilon_{i}$, by definition of $\varepsilon_{i}$. Let $V_{x}$ be an open neighborhood of $\varphi^{\left[t_{x}, 0\right]}(x)$ in $M$ such that

- $\left.\mathcal{H}\right|_{V_{x}}$ is isomorphic to a product foliation,

- each leaf of $\left.\mathcal{H}\right|_{V_{x}}$ intersects $f^{-1}\left(b_{j}\right)$ nontrivially.

Let also $U_{x}$ be a neighborhood of $x$ in $V_{x} \cap \mathcal{M}_{k_{r}}$ for which $U_{x} \cap P_{k_{r}} \simeq U_{x}^{1} \times U_{x}^{2}$, where

- $U_{x}^{1}$ is a neighborhood of $x$ in the leaf $H_{x}$ of $\mathcal{H}$ through $x$,

- $U_{x}^{2}$ is an submanifold containing $x$ whose projection onto the leaf space of $\left.\mathcal{H}\right|_{V_{x}}$ is an embedding.

Existence of $U_{x}$ is guaranteed by the fact that $\mathcal{H}$ is tangent to $P_{k_{r}}-\Sigma_{f}$. Then $\mathcal{S}\left(U_{x} \cap P_{k_{r}}\right) \cap V_{x}$ is an embedded submanifold (it is isomorphic to $U_{x}^{2} \times \mathbb{R}$ ). Now, since $b U_{r} \cap P_{k_{r}} \cap L_{j}$ is compact, it is covered by a finite number of $U_{x}$ 's, say by $U_{x_{1}}, \ldots, U_{x_{n}}$. Moreover, we may assume that for each $1 \leq \ell \leq n$, there exists a relatively compact refinement $V_{\ell}$ of $U_{x_{\ell}}$, such that $\cup_{\ell} V_{\ell}$ also cover $b U_{r} \cap P_{k_{r}} \cap L_{j}$. Then the compact sets $\mathcal{S}\left(\overline{V_{\ell}} \cap P_{k_{r}}\right) \cap L_{j}, \ell=1, \ldots, n$ and $U_{r} \cap P_{k_{r}}$ cover $N_{r}$, and are contained in the submanifolds $\mathcal{S}\left(U_{x_{\ell}} \cap P_{k_{r}}\right) \cap V_{x_{\ell}}$, $\ell=1, \ldots, n$ and $P_{k_{r}}$ respectively. Because $P_{k_{r}}$ is transverse to $\mathcal{F}$, and because $\mathcal{H}$ is tangent to $\mathcal{F}$, the submanifold $\mathcal{S}\left(U_{x_{\ell}} \cap P_{k_{r}}\right) \cap V_{x_{\ell}}$ is transverse to $\mathcal{F}$ as well (since $U_{x}^{2}$ is transverse to $\mathcal{F}$ ). Moreover, by construction, the submanifolds $\mathcal{S}\left(U_{x_{\ell}} \cap P_{k_{r}}\right) \cap V_{x_{\ell}}$ and $P_{k_{r}}$ have codimension at least one.

It follows from Lemma 6.5 and Lemma 6.3 that the stable set of $L_{j} \cap \Sigma_{f}$ with respect to $\left.\left(\nabla_{\mathcal{F}} f\right)\right|_{L_{j}}$ is contained in a finite union of compact subsets of embedded submanifolds intersecting $\mathcal{F}$ transversely, and having their codimension at least equal to one. We can thus use Theorem 1.13 and Remark 1.14 to conclude.

End of the proof of Theorem 6.1.

\section{Examples of uniformely open foliated manifolds.}

The very first class of examples of uniformely open foliated manifolds consists of the products $(M, \mathcal{F}) \times \mathbb{R}$. Let $f$ be any positive, proper, $\mathcal{F}_{-}$ generic function on $(M, \mathcal{F})$ (such a function always exists). The function 
$g: M \times \mathbb{R} \rightarrow \mathbb{R}:(x, t) \mapsto f(x)+t^{2}$ has no leafwise local maxima, and satisfies the hypotheses of Definition 0.3. As implied by the following theorem due to Palmeira, foliations of the type $(M, \mathcal{F}) \times \mathbb{R}$ include an important class of plane foliations (i.e., foliations whose leaves are diffeomorphic to some Euclidean space).

Theorem 7.1 ([19]). If $\mathcal{F}$ is a transversely orientable plane foliation on an orientable $n$-dimensional manifold $M$ (with $n \geq 3$ ), with finitely generated fundamental group, such that all leaves are closed, then there exists a two dimensional surface $S$ and a plane foliation $\mathcal{F}_{0}$ on $S$ such that $\mathcal{F}$ is conjugate to the product of $\mathcal{F}_{0}$ with $\mathbb{R}^{n-2}$.

Another class of examples is described in the following lemma.

Lemma 7.2. Let $\pi: M \rightarrow B$ be a locally trivial fibration with compact fiber $L$ and open base, and let $\mathcal{F}$ be a foliation on $M$ that is transverse to the fibers of $\pi$, in the sense that $T_{x} \mathcal{F}+\operatorname{Ker} \pi_{*_{x}}=T_{x} M$ for all $x$ in $M$. Then the foliated manifold $(M, \mathcal{F})$ is uniformely open.

Proof. Let $g: B \rightarrow[0, \infty)$ be a proper Morse function without local maxima. It is sufficient to prove that if $f: M \rightarrow[0, \infty)$ is sufficiently close to $\pi^{*} g$ in the fine $C^{\infty}$ topology, then $f$ is proper and has no leafwise local maxima. It is easy to see that a function that is $C^{0}$-close to a proper function is proper as well. Let $\left\{x^{j} ; j \geq 1\right\}$ be the set of critical points of $g$. For every $j$, let $x_{1}^{j}, \ldots, x_{k}^{j}$ be local coordinates about $x^{j}$, defined on a neighborhood $U^{j}$ of $x^{j}$ in $B$, for which

$$
g\left(x_{1}^{j}, \ldots, x_{k}^{j}\right)=\left(x_{1}^{j}\right)^{2}+g\left(0, x_{2}^{j}, \ldots, x_{k}^{j}\right) .
$$

Assume also that $U^{j}$ is small enough to guarantee existence of a trivialization $\phi: \pi^{-1}\left(U^{j}\right) \rightarrow U^{j} \times L$, with the property that the image of each local sections $\sigma_{\ell}^{j}: U^{j} \rightarrow \pi^{-1}\left(U^{j}\right): x \mapsto \phi^{-1}(x, \ell), \ell \in L$ is contained in a leaf of $\mathcal{F}$. A function $f$ whose 2-jet is sufficiently close to that of $\pi^{*} g$ satisfies the following properties.

- The leafwise critical points of $f$ are all contained in $\pi^{-1}\left(\cup_{j} U^{j}\right)$.

- For all $j \geq 1$, and for all $\ell$ in $L$, the second derivative of $f \circ \sigma_{\ell}^{j}$ in the direction of $x_{1}^{j}$ is strictly positive on $U^{j}$. 
Then, if $y$ is a leafwise critical point of $f$, the foliated second differential of $f$ at $y$ may not be negative definite, that is, $y$ may not be a leafwise local maximum of $f$.

Remark 7.3. It is not know to us whether locally trivial fibrations with open fibers are always uniformely open foliated manifolds. Nevertheless, such foliated manifolds satisfy the conclusion of Theorem 0.4, as implies Theorem 0.1 (cf. $[1,2])$.

We will now describe a general procedure that allows one to construct uniformely open foliated manifolds. We need to recall the notion of Novikov component of a codimension one foliation $\mathcal{F}$ on a closed manifold $M$.

Definition 7.4 ([18]). Two points $x$ and $y$ in $M$ are said to be equivalent with respect to $\mathcal{F}$ if either $F_{x}=F_{y}$, or the foliation $\mathcal{F}$ admits a closed transversal that contains both $x$ and $y$.

It is not difficult to verify that this defines an equivalence relation on $M$.

Definition 7.5. A Novikov component of the foliation $\mathcal{F}$ is an equivalence class for this equivalence relation.

Theorem 7.6 ([18]). A Novikov component is either a compact leaf or an open submanifold whose boundary is a finite union of compact leaves which are themselves Novikov components.

Example 7.7. The Reeb foliation on $S^{3}$ has three Novikov components. One of them is the torus leaf. The other two are the two open solid tori bounded by the torus leaf.

Remark 7.8. A transversely orientable codimension one foliation with one Novikov component may have compact (necessarily nonseparating) leaves. Such compact leaves may admit closed transversal that do not pass through all the leaves. (See M.P. Muller's example [16].)

The following result is due to Ferry and Wasserman.

Theorem $7.9([6])$. For a codimension one foliation $\mathcal{F}$ on a closed manifold $M$, the following statements are equivalent.

- $\mathcal{F}$ has one Novikov component. 
- There exists an $\mathcal{F}$-generic function $f: M \rightarrow \mathbb{R}$ with no degenerate leafwise critical points.

Consider now a codimension one foliation $\mathcal{F}$ with one Novikov component on a closed $(n+1)$-dimensional manifold $M$. Let $f: M \rightarrow \mathbb{R}$ be an $\mathcal{F}$-generic function without degenerate leafwise critical points. Let $\Gamma \subset M$ be the set of leafwise local maxima of $f$. Notice that since $f$ has no degenerate leafwise critical points, its singular locus (Definition 3.2) is necessarily transverse to $\mathcal{F}$ (cf. Section 3.1 or [6]). It is therefore a finite union of embedded circles intersecting $\mathcal{F}$ transversely; the set $\Gamma$ is the union of some of them.

Lemma 7.10. The foliated manifold $\left(M^{\prime}=M-\Gamma, \mathcal{F}^{\prime}=\left.\mathcal{F}\right|_{M-\Gamma}\right)$ is uniformely open.

Proof. We already have a bounded below $\mathcal{F}$-generic function $\left.f\right|_{M^{\prime}}$ on $M^{\prime}$ with no leafwise local maxima. The only thing that needs to be done is to modify $\left.f\right|_{M^{\prime}}$ so as to make it proper. Take a tubular neighborhood $U$ of $\Gamma$ in $M$. The set $U$ is the image of an embedding $e: E \rightarrow M$, defined on the total space of a rank- $n$ vector bundle $p: E \rightarrow \Gamma$, such that $e \circ s=\operatorname{Id}_{\Gamma}$, where $s: \Gamma \rightarrow E$ is the zero section. Since $\Gamma$ is transverse to $\mathcal{F}$, we may assume, without loss of generality, that the foliation $\left.\mathcal{F}\right|_{U}$ corresponds, under the map $e$, to the foliation of $E$ by the fibers of $p$. Such a tubular neighborhood is called hereafter a foliated tubular neighborhood. Let $g$ be a Riemannian metric on the bundle $E$. Let $\theta: \mathbb{R} \rightarrow[0,1]$ be a function with compact support whose only critical value, aside from 0 , is a global maximum achieved at the point 0 . Consider the function

$$
f^{\prime}: M^{\prime} \rightarrow \mathbb{R}: x \mapsto \begin{cases}f(x)+\frac{\theta\left(g\left(e^{-1} x, e^{-1} x\right)\right)}{g\left(e^{-1} x, e^{-1} x\right)} & \text { for } x \text { in } U-\Gamma \\ f(x) & \text { otherwise. }\end{cases}
$$

The function $f^{\prime}$ is proper and bounded below. Moreover, provided $U$ is small enough, its leafwise critical points are exactly those of $\left.f\right|_{M^{\prime}}$. Thus $f^{\prime}$ has no leafwise local maxima.

Remark 7.11. Making $\left.f\right|_{M-\Gamma}$ proper requires the set $\Gamma$ of leafwise local maxima of $f$ to be a union of circles. If on the contrary, $\Gamma$ has a line segment as one of its connected components, as might be the case if the function $f$ had degenerate leafwise critical points, it would not be possible to make $\left.f\right|_{M-\bar{\Gamma}}$ proper without creating leafwise local maxima. 
Remark 7.12. It is a classical result due to Rummler and Sullivan that a transversely orientable codimension one foliation has one Novikov component if and only if it is geometrically taut, that is, if and only if $M$ admits a Riemannian metric with respect to which all the leaves of $\mathcal{F}$ are minimal submanifolds (a proof of this result can be found in [4]).

As was suggested to us by $\mathrm{T}$. Tsuboi, interesting foliations with one Novikov component are obtained by identifying deleted tubular neighborhoods of closed transversals (idea originally due to M. Hirsch). More precisely, let $M$ be an $(n+1)$-dimensional manifold endowed with a codimension one foliation $\mathcal{F}$. Let $c_{1}, c_{2}: S^{1} \rightarrow M$ be disjoint embeddings transverse to $\mathcal{F}$. Suppose that the normal bundle of $c_{i}\left(S^{1}\right)$ is trivial (which it is when the foliated manifold $(M, \mathcal{F})$ is orientable). Consider disjoint foliated tubular neighborhood $U_{1}$ and $U_{2}$ of $c_{1}\left(S^{1}\right)$ and $c_{2}\left(S^{1}\right)$ respectively. The set $U_{i}$ is the image of an embedding $e_{i}: S^{1} \times \mathbb{R}^{n} \rightarrow M$ that coincides with $c_{i}$ on $S^{1} \times\{0\}$, and that maps the "vertical" foliation of $S^{1} \times \mathbb{R}^{n}$ isomorphically onto the foliation $\left.\mathcal{F}\right|_{U_{i}}$. The fiberwise connected sum along the closed transversals $c_{1}$ and $c_{2}$, denoted hereafter by $(M, \mathcal{F})_{c_{1} \# c_{2}}$, is defined to be the foliated manifold obtained by removing the two curves $c_{1}\left(S^{1}\right)$ and $c_{2}\left(S^{1}\right)$ from $M$ and identifying $U_{1}-c_{1}\left(S^{1}\right)$ with $U_{2}-c_{2}\left(S^{1}\right)$ by means of the map

$$
U_{1}-c_{1}\left(S^{1}\right) \rightarrow U_{2}-c_{2}\left(S^{1}\right): e_{1}(t, x) \mapsto e_{2}\left(t, \frac{x}{\|x\|^{2}}\right)
$$

where $t \in S^{1}$ and $x \in \mathbb{R}^{n}$. If $\mathcal{F}$ has one Novikov component, so does $(M, \mathcal{F})_{c_{1} \# c_{2}}$.

Consider, for instance, a manifold of the type $M=S^{1} \times F$, where $F$ is some $n$-dimensional manifold. Let $\pi$ denote the natural projection $M \rightarrow S^{1}$, and let $\mathcal{F}$ denote the (trivial) foliation of $M$ by the fibers of $\pi$. Let also $c_{1}, c_{2}$ : $S^{1} \rightarrow M$ be disjoint embeddings such that $\pi \circ c_{1}$ coincides with $\operatorname{Id}_{S^{1}}$ and $\pi \circ c_{2}$ coincides with the double cover $e^{i \theta} \mapsto e^{i 2 \theta}$ (alternatively, one could suppose that $\pi \circ c_{1}$ and $\pi \circ c_{2}$ are different covers of $S^{1}$ of type $\left.e^{i \theta} \mapsto e^{i n \theta}\right)$. Then the foliated manifold $(M, \mathcal{F})_{c_{1} \# c_{2}}$ has one Novikov component, and dense leaves. Indeed, a leaf of the connected sum corresponds to an equivalence class for the equivalence relation on $S^{1}$ generated by $e^{i \theta} \sim e^{i 2 \theta}$. The uniformely open foliated manifold obtained by removing from $M$ the set of closed curves along which some given $\mathcal{F}$-generic function without degenerate leafwise critical points achieves leafwise local maxima has dense leaves as well. Besides, if $F$ is an open manifold, it is not necessary to remove anything from $(M, \mathcal{F})_{c_{1} \# c_{2}}$ to achieve uniform openness. 
Lemma 7.13. If the manifold $F$ is open, then the fiberwise connected sum $(M, \mathcal{F})_{c_{1} \# c_{2}}$ is uniformely open.

Proof. Let $f: F \rightarrow[0, \infty)$ be a proper Morse function without local maxima. Let $a$ be a noncritical value of $f$. Let $\gamma: S^{1} \rightarrow F$ be an injective map whose image is contained in the level set $f^{-1}(a)$. Let $q$ be a point in $f^{-1}(a)-\gamma\left(S^{1}\right)$. Suppose that the closed transversal $c_{1}$ coincides with the map $S^{1} \rightarrow M: t \mapsto$ $(t, q)$, and that $c_{2}$ coincides with the map $S^{1} \rightarrow M: t \mapsto(2 t, \gamma(t))$. Suppose also that on the tubular neighborhoods $U_{1}$ and $U_{2}$ of $c_{1}$ and $c_{2}$ respectively along which the identification is performed, the function $p^{*} f$ corresponds to some function of the following type :

$$
S^{1} \times \mathbb{R}^{n} \rightarrow \mathbb{R}:\left(t, x_{1}, \ldots, x_{n}\right) \mapsto a+h\left(x_{n}\right),
$$

where $p$ denotes the projection $M \rightarrow F$ and where $h: \mathbb{R} \rightarrow \mathbb{R}$ is an embedding. The function $\left.p^{*} f\right|_{M-\left(c_{1}\left(S^{1}\right) \cup c_{2}\left(S^{1}\right)\right)}$ yields a proper function $f^{\prime}: M_{c_{1} \# c_{2}} \rightarrow[0, \infty)$ that coincides with $p^{*} f$ on $M-\left(U_{1} \cup U_{2}\right)$, and whose critical locus in $\left(U_{1}-c_{1}\left(S^{1}\right)\right) \simeq\left(U_{2}-c_{2}\left(S^{1}\right)\right)$ is made of two disjoint transverse closed curves, consisting of leafwise critical points of leafwise index $n-1$ and 1 respectively. Figure 1 shows how to construct $f^{\prime}$ on the connected sum of a fiber in the tubular neighborhood $U_{1}$ with the corresponding fiber in the tubular neighborhood $U_{2}$.

\section{Application to Poisson geometry.}

Let us recall that a regular Poisson manifold can be described as a foliated space $(M, \mathcal{F})$ endowed with a leafwise symplectic structure, that is, a section of the second exterior power of the cotangent bundle $T^{*} \mathcal{F}$ of the foliation $\mathcal{F}$, whose restriction to each leaf of $\mathcal{F}$ is a symplectic form (cf. [5]). The question of existence of such a structure on a given foliated manifold as been approached in a previous paper [2] (see also [1]), where examples of foliations are presented that do not support any leafwise symplectic structure although the obvious obstructions vanish. On the other hand, as explained below, a leafwise symplectic structure on the foliated manifold $(M, \mathcal{F})$ is a solution of a certain open, foliated invariant differential relation.

Consider a foliated manifold $(M, \mathcal{F})$. A section of the bundle $\Lambda^{k} T^{*} \mathcal{F}$ is called a tangential differential $k$-form. A tangential differential 2-form $\alpha$ is said to be nondegenerate if for every point $x$ in $M$, the skewsymmetric 


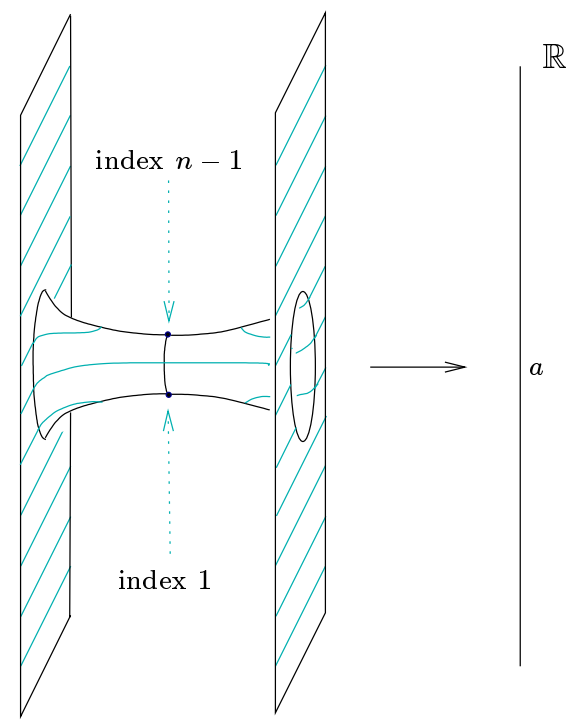

Figure 1: The function $f^{\prime}$ on the connected sum of two corresponding fibers.

bilinear form $\alpha(x)$ on $T_{x} \mathcal{F}$ is nondegenerate. The usual exterior differential restricts naturally into a map $d_{\mathcal{F}}: \Gamma\left(\Lambda^{\star} T^{*} \mathcal{F}\right) \rightarrow \Gamma\left(\Lambda^{\star+1} T^{*} \mathcal{F}\right)($ cf. [11]). The corresponding cohomology

$$
H^{\star}(\mathcal{F})=\frac{\left\{d_{\mathcal{F}}-\text { closed } \star-\text { forms }\right\}}{\left\{d_{\mathcal{F}}-\text { exact } \star-\text { forms }\right\}},
$$

is called the tangential de Rham cohomology of the foliated manifold $(M, \mathcal{F})$. There is a natural affine fibration $L:\left(T^{*} \mathcal{F}\right)^{1} \rightarrow \Lambda^{2} T^{*} \mathcal{F}$ defined by

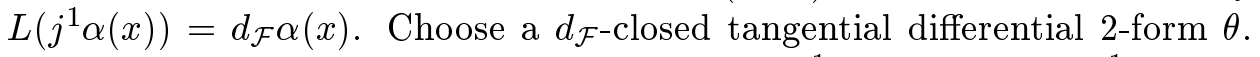
Define $\Omega_{\theta}$ to be the differential relation $\Omega_{\theta}=\left\{j^{1} \alpha(x) ; \theta(x)+L\left(j^{1} \alpha(x)\right)\right.$ is nondegenerate $\}$. A solution of $\Omega_{\theta}$ is thus a tangential differential 1-form $\alpha$ such that $d_{\mathcal{F}} \alpha+\theta$ is a leafwise symplectic structure belonging to the same tangential de Rham cohomology class as $\theta$. Thus, the set of solutions, modulo the set of $d_{\mathcal{F}}$-closed 1-forms, parameterizes the set of leafwise symplectic structures lying in the class $[\theta]$. On the other hand, since the map $L$ is an affine fibration, the space of sections of $\Omega_{\theta}$ is weakly homotopy equivalent to the space of leafwise nondegenerate 2 -forms.

Proposition 8.1 ([7]). The relation $\Omega_{\theta}$ is open and invariant under foliated isotopies. 
Proof. That $\Omega_{\theta}$ is open follows directly from its definition. The second assertion relies on considering the "right" lift for isotopies, that is :

$$
\varphi_{t} \cdot \alpha=\varphi_{t}^{*} \alpha+\int_{0}^{t} \varphi_{s}^{*}\left(i\left(X_{s}\right) \theta\right) d s
$$

where $\alpha$ is an element of $T^{*} \mathcal{F}$, and where $X_{t}$ denotes the time-dependent vector field associated to the isotopy $\varphi_{t}$. If $\alpha$ is a tangential differential 1-form for which the tangential differential 2-form $d_{\mathcal{F}} \alpha+\theta$ is leafwise nondegenerate, then $\varphi_{t}^{*}\left(d_{\mathcal{F}} \alpha+\theta\right)$ is leafwise nondegenerate as well (while the form $\varphi_{t}^{*}\left(d_{\mathcal{F}} \alpha\right)+\theta$ might very well be leafwise degenerate), and

$$
\begin{aligned}
\varphi_{t}^{*}\left(d_{\mathcal{F}} \alpha+\theta\right) & =d_{\mathcal{F}} \varphi_{t}^{*} \alpha+\theta+\left(\varphi_{t}^{*} \theta-\theta\right) \\
& =d_{\mathcal{F}} \varphi_{t}^{*} \alpha+\theta+\left.\int_{0}^{t} \frac{d}{d s} \varphi_{s}^{*} \theta\right|_{s} d s \\
& =d_{\mathcal{F}} \varphi_{t}^{*} \alpha+\theta+\int_{0}^{t} \varphi_{s}^{*} \mathcal{L}_{X_{s}} \theta d s \\
& =d_{\mathcal{F}} \varphi_{t}^{*} \alpha+\theta+\int_{0}^{t} \varphi_{s}^{*} d_{\mathcal{F}} i\left(X_{s}\right) \theta d s \\
& =d_{\mathcal{F}}\left(\varphi_{t}^{*} \alpha+\int_{0}^{t} \varphi_{s}^{*} i\left(X_{s}\right) \theta d s\right)+\theta
\end{aligned}
$$

Thus, Theorem 0.4 applies to the relation $\Omega_{\theta}$.

Theorem 8.2. Let $(M, \mathcal{F})$ be a uniformely open foliated manifold. Given a $d_{\mathcal{F}}$-closed tangential differential 2-form $\theta$, any family $\beta_{s}, s \in[0,1]^{p}$ of leafwise nondegenerate 2-forms is homotopic to a family of leafwise symplectic structures cohomologous to $\theta$. Moreover, if $\beta_{s}$ is already symplectic and cohomologous to $\theta$ for $s$ in $\partial\left([0,1]^{p}\right)$, the homotopy may be chosen to be stationary for those parameters. Equivalently, the inclusion of the space of leafwise symplectic structures cohomologous to $\theta$ into the space of leafwise nondegenerate 2-forms is a weak homotopy equivalence

Theorem 8.2 implies the following existence and uniqueness result for leafwise symplectic structures.

Corollary 8.3. On a uniformely open foliated manifold any leafwise nondegenerate 2-form can be deformed into a leafwise symplectic form (with 
prescribed tangential de Rham cohomology class). Moreover, if two cohomologous leafwise symplectic structures can be joined by a path of leafwise nondegenerate 2-forms, they can also be joined by a path of cohomologous leafwise symplectic forms.

\section{References.}

[1] M. Bertelson, Foliations associated to regular Poisson structures, Ph.D. Thesis, Stanford University, June 2000.

[2] M. Bertelson, Foliations associated to regular Poisson structures, Commun. Contemp. Math., 3(3) (2001), 441-456.

[3] J.M. Boardman, Singularities of differentiable maps, Inst. Hautes Études Sci. Publ. Math., 33 (1967), 21-57.

[4] A. Candel and L. Conlon, Foliations, I, Graduate Studies in Mathematics, 23, American Mathematical Society, Providence, RI, 2000.

[5] A. Cannas da Silva and A. Weinstein, Geometric Models for Noncommutative Algebras, Berkeley Mathematics Lecture Notes Series, American Mathematical Society, 1999.

[6] S. Ferry and A. Wasserman, Morse theory for codimension-one foliations, Trans. Amer. Math. Soc., 298(1) (1986), 227-240.

[7] E. Giroux, Flexibilité en géométrie symplectique, d'après Mikhael Gromov, Unpublished lecture notes, 1993.

[8] M. Golubitsky and V. Guillemin, Stable mappings and their singularities, Graduate Texts in Mathematics, 14, Springer-Verlag, New YorkHeidelberg, 1973.

[9] M. Gromov, Stable mappings of foliations into manifolds, Izv. Akad. Nauk SSSR Ser. Mat., 33 (1969), 707-734.

[10] M. Gromov, Partial differential relations, Ergebnisse der Mathematik und ihrer Grenzgebiete (3) [Results in Mathematics and Related Areas (3)], 9, Springer-Verlag, Berlin-New York, 1986. 
[11] G. Hector, E. Macías and M. Saralegi, Lemme de Moser feuilleté et classification des variétés de Poisson régulières (French), [A foliated version of Moser's lemma and classification of regular Poisson manifolds], Publ. Mat., 33(3) (1989), 423-430.

[12] K. Igusa, Higher singularities of smooth functions are unnecessary, Ann. of Math. (2), 119 (1984), 1-58.

[13] J. Martinet, Singularities of smooth functions and maps, Translated from the French by Carl P. Simon. London Mathematical Society Lecture Note Series, 58, Cambridge University Press, Cambridge-New York, 1982.

[14] J.N. Mather, On Thom-Boardman singularities. Dynamical systems, (Proc. Sympos., Univ. Bahia, Salvador, 1971), 233-248, Academic Press, New York, 1973.

[15] J.N. Mather, Stability of $C^{\infty}$ mappings, III. Finitely determined mapgerms, Inst. Hautes Studes Sci. Publ. Math., 35 (1968), 279-308.

[16] M.P. Muller, Sur les composantes de Novikov des feuilletages, Topology, 19 (1980), 199-201.

[17] J. Milnor, Morse theory. Based on lecture notes by M. Spivak and R. Wells., Annals of Mathematics Studies, 51, Princeton University Press, Princeton, N.J. 1963.

[18] S.P. Novikov, Topology of foliations, Trans. Moscow Math. Soc., 14 (1965), 268-304.

[19] C.F.B. Palmeira, Open manifolds foliated by planes, Ann. Math. (2), 107(1) (1978), 109-131.

[20] C.H. Taubes, The Seiberg-Witten invariants and symplectic forms, Math. Res. Lett., 1(6) (1994), 809-822. 
[21] H. Whitney, Elementary structure of real algebraic varieties, Ann. of Math. (2), 66 (1957), 545-556.

DÉPartement de MathÉmatiques

Université LiBRe de Bruxelles

Boulevard DU TRIOMPHE

B-1050 BRUXELLES

E-mail address: mbertels@ulb.ac.be 
